\title{
Fine-structure infrared lines from the Cassiopeia A knots
}

\author{
D. Docenko ${ }^{1,2}$ and R. A. Sunyaev ${ }^{1,3}$ \\ 1 Max Planck Institute for Astrophysics, Karl-Schwarzschild-Str. 1, 85741 Garching, Germany \\ 2 Institute of Astronomy, University of Latvia, Raiņa bulvāris 19, Riga LV-1586, Latvia \\ e-mail: dima@latnet.lv \\ 3 Space Research Institute, Russian Academy of Sciences, Profsoyuznaya 84/32, 117997 Moscow, Russia
}

Received 10 June 2008 / Accepted 13 October 2009

\begin{abstract}
Aims. Archival observations of infrared fine-structure lines of the young Galactic supernova remnant Cassiopeia A allow us to test existing models and determine the physical parameters of various regions of the fast-moving knots, which are metal-dominated clouds of material ejected by the supernova explosion.

Methods. The fluxes of far-infrared [OI] and [O III] lines are extracted from previously unpublished archival ISO data. The archival Spitzer data are used to determine the fluxes of the $\mathrm{O}, \mathrm{Ne}, \mathrm{Si}, \mathrm{S}, \mathrm{Ar}$, and $\mathrm{Fe}$ ion fine-structure lines originating in the fast-moving knots. The ratios of these line fluxes are used as plasma diagnostics. We also determine the infrared line flux ratios with respect to the optical [O III] $5007 \AA$ Aine in the knots with previously measured reddening. Additionally, we analyze several optical and near-infrared observations of the fast-moving knots to obtain clearer insight into the post-shock photoionized region structure.

Results. We show that the infrared oxygen line flux predictions of all existing theoretical models are correct only to within a factor of a several. Comparison of the model predictions shows that to reproduce the observations it is essential to include the effects of the electron conductivity and dust. Detailed analysis of the diagnostic line flux ratios of various ions allows us to qualitatively confirm the general model of fast-moving knot emission and determine observationally for the first time the physical conditions in the photoionized region after the shock. We infer from the [O III] line flux ratios that the pre-shock cloud densities are higher than assumed in existing theoretical models and most probably correspond to several hundred particles per $\mathrm{cm}^{3}$. We also determine the Cas A luminosity in the infrared continuum and lines. We show that accounting for the charge exchange processes in the post-shock photoionized region allows us to reproduce most of the relevant spectral line ratios even in the frame of a single-temperature model of this region. We also estimate its plasma parameters, thickness, and carbon abundance.
\end{abstract}

Key words. ISM: supernova remnants - infrared: ISM - atomic processes

\section{Introduction}

The young Galactic supernova remnant Cassiopeia A (Cas A) is one of only a few objects where the inner composition of the supernova progenitor may be studied directly, as the explosion ejecta have not yet mixed with the surrounding medium. In places where these ejecta are being crossed by the reverse shock wave, they are observable as compact knots emitting optical forbidden lines of ions of oxygen, sulfur, argon, and other elements. Because of their high density, these ejecta knots are not strongly decelerated by the reverse shock and, as a result, have high proper motions that was the reason to name them as the fastmoving knots (FMKs). Studies of Peimbert \& van den Bergh (1971) and Chevalier \& Kirshner $(1978,1979)$ firmly established that the FMKs are an observational manifestation of the supernova ejecta.

In FMKs, we therefore deal with a very unusual situation in astrophysics, where oxygen comprises $80 \%-90 \%$ of all atoms in the plasma, other components being mostly $\mathrm{Ne}, \mathrm{Si}, \mathrm{S}, \mathrm{Ar}$, and $\mathrm{Fe}$. The hydrogen and helium abundances are, in contrast, understood to be negligibly low. Optical observations have shown that some knots consist even of almost pure oxygen (so-called [O III] filament, Chevalier \& Kirshner 1979).

Theoretical interpretation of the optical observations has proven that the plasma heated up to temperatures of several million degrees is rapidly cooling because of its high density and the anomalous chemical composition of the gas. At the same time, the ionization degree lags behind the rapid cooling rate. This results in a unique situation where atoms with strongly different ionization degrees coexist at low temperatures from $10^{4} \mathrm{~K}$ to several hundreds Kelvin. Because of this, as we now show, the lines of highly-charged ions, such as [Si X] and [Ar V], originate at temperatures of about $10^{4} \mathrm{~K}$ and lower ${ }^{1}$.

Traditionally, theoretical models of fast-moving knot emission were compared only with optical line observations. Since there is only a very limited number of diagnostic line ratios in the optical spectrum, the FMK theoretical models are difficult to both construct and compare with optical observations. The most well-known models are those constructed by Itoh (1981a,b), Borkowski \& Shull (1990), and Sutherland \& Dopita (1995).

Docenko \& Sunyaev (2008) illustrated one way of constraining the theoretical models using prospective observations of optical and near-infrared recombination lines of highly-charged oxygen ions.

In this paper, we demonstrate how existing archival infrared observations can discriminate between existing models and place strong constraints on the construction of future ones. We compare the model predictions of the ratios of far-infrared

1 The [Si X] line has also been detected in spectra of several active galactic nuclei (Thompson 1996; Riffel et al. 2006), where it is apparently formed in the photoionized region. Even so, the models predict line-forming region temperatures of around $10^{5} \mathrm{~K}$ (Ferguson et al. 1997). 
A\&A 509, A59 (2010)

Table 1. Far-infrared line flux ratios to the dereddened $5007 \AA$ line as derived from different theoretical models and observations. (See text for details).

\begin{tabular}{|c|c|c|c|c|c|c|c|}
\hline & \multirow{2}{*}{$\lambda, \mu \mathrm{m}$} & \multicolumn{5}{|c|}{$\overline{I / I(5007)}$} & \multirow{2}{*}{$\begin{array}{l}f_{\mathrm{PIR}} \\
\text { SD-200 }\end{array}$} \\
\hline & & I-H & BS-F & BS-DC & SD-200* & Observed $^{* *}$ & \\
\hline [O IV] & 25.91 & 0.0051 & 5.0 & 0.53 & 8.1 & 0.20 & 0.995 \\
\hline [O III] & 51.81 & 0.031 & 1.34 & 0.72 & 0.67 & 0.25 & 0.999 \\
\hline [O I] & 63.19 & 1.30 & 170 & 12 & - & 0.07 & - \\
\hline [O III] & 88.36 & 0.028 & 0.22 & 0.11 & 0.55 & 0.10 & 0.999 \\
\hline$[\mathrm{OI}]$ & 145.5 & 0.038 & 7.8 & 0.75 & - & $<0.0024$ & - \\
\hline
\end{tabular}

* Derived in Sects. 2.1 and 2.2; ** obtained in Sects. 3.1 and 3.2.1.

Notes: the last column lists contribution to the line flux in the SD-200 model arising from the photoionized region (PIR) before the shock. Derivation of the observed line flux ratio values is described in Sect. 3. The model-predicted [O I] lines are sensitive to the pre-shock ion number density in the model, see Sect. 4.1.2.

(FIR) fine-structure oxygen line fluxes to the flux in the optical [O III] $5007 \AA$ line with their observational values, and show that all of the predictions are incorrect by a factor of several for one or several flux ratios. It should be remembered, however, that the theoretical models predict the deviations of ionic abundances from their collisional equilibrium values by several orders of magnitude. Therefore, inconsistencies of the observed order suggest that some corrections should be applied to the existing models, while the general picture described in the models is entirely correct.

The infrared fine-structure line flux ratios of other element (in addition to oxygen) ions are currently the most reliable tool available for direct studies of the physical parameters in the FMKs. We attempted to estimate these parameters using available observational data. As a result, parameters of the post-shock photoionized region were estimated observationally for the first time, confirming qualitatively the predictions of the theoretical models, but again highlighting some quantitative differences.

The FIR lines will provide even more important probe in the future, as several far-infrared observatories, such as the Herschel Space Observatory and SOFIA, begin their operation. These telescopes will provide far higher quality FIR data ofhigher sensitivity and angular resolution, allowing us to constrain the FMK models more tightly. Results of this present study are also important to understand the small-scale structure of other oxygen-rich supernova remnants, such as Puppis A, N132D, and G292+1.8.

The paper structure is the following. In the next section, we describe briefly the existing theoretical models of the FMKs and estimate oxygen infrared line fluxes in one of these models. Section 3 describes the archival infrared observations of Cas A and their analysis. In Sect. 4, we estimate the physical conditions and abundances in different regions of FMKs from the observed line flux ratios. We also discuss these results and compare them with values predicted by the models. In Sect. 5, we analyze spectral line ratios to obtain a consistent model of the post-shock photoionized region. Section 6 is devoted to the predictions of the mid- and far-infrared recombination line intensities. Finally, in Sect. 7 we present our conclusions.

\section{Theoretical models of fast-moving knots}

The first detailed model of fast-moving knot emission, describing it as originating from the passage of a shock through the pure oxygen medium, was constructed by Itoh $(1981 \mathrm{a}, \mathrm{b})$. The predictions of his model $\mathrm{H}$ were compared with measurements of the [O III] filament optical spectrum in the northern part of the Cas A supernova remnant (SNR) shell, and were found to reproduce all four measured oxygen [O I], [O II], and [O III] optical line flux

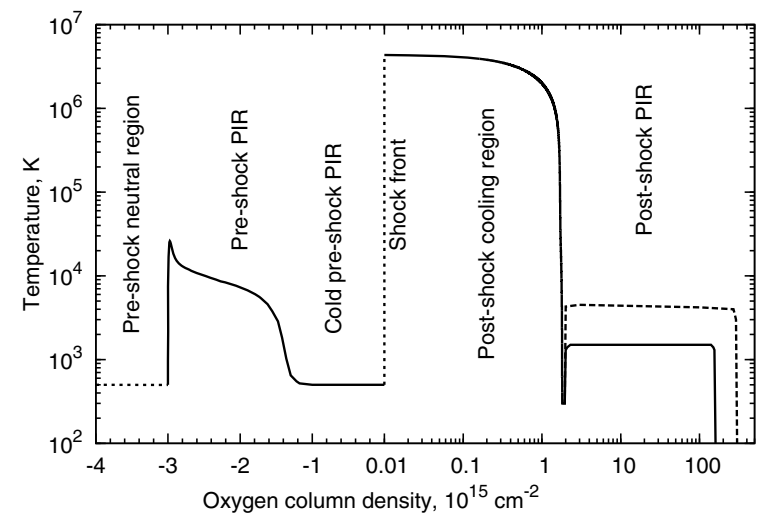

Fig. 1. Schematic representation of the FMK temperature structure, induced by its interaction with the reverse shock. Negative column densities corresponding to the pre-shock regions are in linear scale; positive (post-shock) column densities are on a logarithmic scale. The post-shock photoionized region (PIR) parameters are given as derived in Sect. 5 (solid line) and according to the Borkowski \& Shull (1990) models (dashed line). Shock is moving to the left. Because of the FMK high density, the reverse shock in it has slowed down to about $200 \mathrm{~km} \mathrm{~s}^{-1}$.

ratios to within a factor of two. The infrared line ratios to the [O III] $5007 \AA$ line $e^{2}$ for this theoretical model (denoted I-H) and other models described below are given in Table 1 .

In this and other theoretical models, the rapidly cooling region just after the shock front produces high ionizing radiation flux that in its turn produces two photoionized regions (PIRs), before and after the shock front (see Fig. 1).

Itoh (1986) found that the model neutral oxygen optical line intensities originating in the PIR after the shock front are much too high compared to the observed spectra of the oxygen-rich supernova remnant Cas A and Puppis A ejecta. He suggested that emission from this region is damped because the region itself is truncated due to some hydrodynamical phenomena. This truncation affects essentially only neutral oxygen lines, as all the other ions have already recombined in the dense post-shock plasma before reaching the photoionized region. This possibly ensures that the $[\mathrm{OI}]$ lines are the least reliable for direct comparison with model predictions, since all existing models are one-dimensional.

Another group of theoretical FMK models was proposed by Borkowski \& Shull (1990). In contrast to the Itoh models,

\footnotetext{
2 The $5007 \AA$ line is three times brighter than the other [O III] doublet component at $4959 \AA$ (e.g., Osterbrock \& Ferland 2006). Note that in some papers the line intensities are compared to the sum of the doublet components.
} 
D. Docenko and R. A. Sunyaev: Fine-structure infrared lines from the Cassiopeia A knots

some of these accounted for electron conductivity, which changed the line fluxes considerably. One more difference is that the Borkowski \& Shull (1990) models do not account for the emission from the PIR before the shock. The models that most accurately describe the Cas A FMK optical spectra are F and DC (respectively, denoted as BS-F and BS-DC in Table 1 and below). We note that only the BS-DC model takes the electron conductivity into account.

Both Itoh (1981a,b) and Borkowski \& Shull (1990) assume that the emission originates in the pure oxygen plasma. However, it was shown by Dopita et al. (1984) that inclusion of other elements in the model significantly changes the plasma thermal structure and emission, especially in the cold PIRs, where a number of fine-structure transitions in ions of other elements can effectively cool plasma to temperatures of about one hundred Kelvin. Following this line of reasoning, another group of the FMK models was published by Sutherland \& Dopita (1995). These models assume that the emission is produced by the interaction of the dense cloud with the external shock, entering the cloud and propagating through it. The expected far-infrared line intensities are not published and we estimate them in the Sects. 2.1 and 2.2 for the model with a $200 \mathrm{~km} \mathrm{~s}^{-1}$ shock speed and pre-shock ion number density of $100 \mathrm{~cm}^{-3}$ (denoted as SD200 in Table 1 and below), which most accurately reproduces the optical spectra of Cas A fast-moving knots, as demonstrated by Sutherland \& Dopita (1995).

Compared to the models of Itoh (1981a,b) and Borkowski \& Shull (1990), the SD-200 model does not include the PIR after the shock front, because it is constructed to describe the FMK optical spectrum and the temperature of this post-shock PIR was estimated to be too low to contribute significantly to the optical line emission.

All described models nevertheless share many similar features (see Fig. 1). In all of them, the plasma after the shock front passage rapidly cools and, at temperatures of $(5-50) \times 10^{3} \mathrm{~K}$, emits the high-ionization lines observable in the visible and nearinfrared spectra (Chevalier \& Kirshner 1978, 1979; Hurford \& Fesen 1996; Gerardy \& Fesen 2001). The thickness of this emitting layer is extremely small (about $10^{10} \mathrm{~cm}$ for the pre-shock ion density of $100 \mathrm{~cm}^{-3}$ ), but because of the high electron density in the cooling material at $T<10^{5} \mathrm{~K}$ (more than $10^{5} \mathrm{~cm}^{-3}$ ), the emission measure is enough to produce or contribute to bright emission lines of highly-charged ionic species, such as [O III] lines near $5000 \AA$.

In all of these models, the visible line emission of weaklyionized ionic species (e.g., [O I] and [O II]) originates in photoionized regions before and after the shock front.

Although all of the theoretical models describe optical spectra relatively well, with discrepancies in relative intensities that do not exceed factors of about two, predictions for the infrared lines differ by several orders of magnitude (see Table 1). It is therefore clear that the models have a lack of constraining power and more diagnostic information in the form of various line flux ratios is needed to determine the true structure of the fast-moving knots. Part of this information may be obtained from the farinfrared line archival observations, and we present this analysis in Sect. 3 below.

\subsection{Infrared lines from the $S D-200$ model}

Because of a lack of published values, we had to make our own estimates of the far-infrared line fluxes and their ratios with respect to the [O III] $5007 \AA$ line in the SD-200 model. Fortunately,

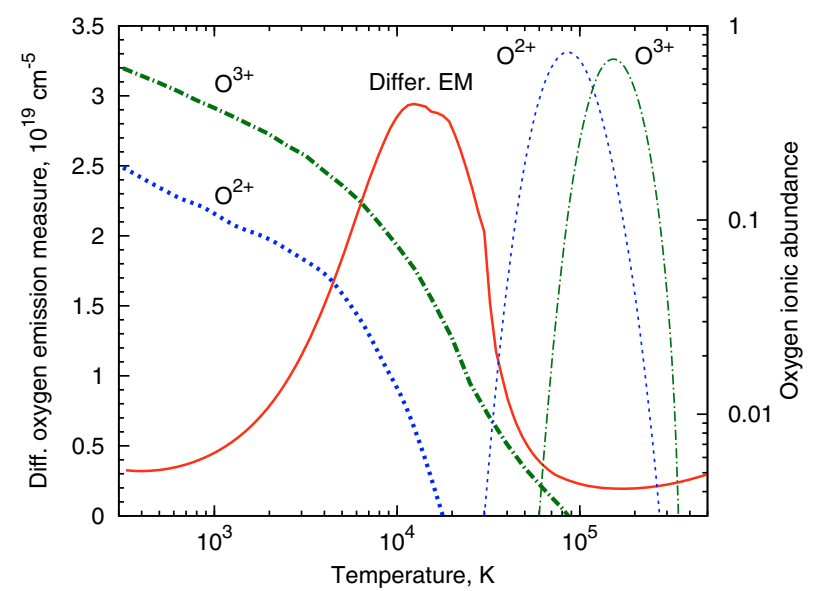

Fig. 2. Comparison of ionic abundances of oxygen ions $\mathrm{O}^{2+}$ and $\mathrm{O}^{3+}$ in the collisional ionization equilibrium (thin curves) and the cooling post-shock region of the SD-200 model (thick curves of the same color). The differential oxygen emission measure per logarithmic temperature interval $\mathrm{d} E_{\mathrm{O}} / \mathrm{d} \ln T_{\mathrm{e}}$ is indicated by the red solid line.

all the data necessary for this calculation - the ionic abundances of all oxygen ions, the temperature and density structure of the post-shock region, and the temperature structure of the preshock photoionization front - are known ${ }^{3}$.

We note that the FMK structure calculations in the SD-200 model are based on the plasma composition that differs slightly from the composition inferred from the X-ray and optical observations. Specifically, it is dominated by $\mathrm{O}(63 \%$ by number $)$ and $\mathrm{Ne}(29 \%)$, and contains minor amounts of C, Mg and Si. In contrast, the observations imply that the majority of the currently bright X-ray and optical plasma is oxygen (about 90\%), other abundant elements being not only $\mathrm{Ne}$, but also $\mathrm{Si}$ and $\mathrm{S}$.

In Fig. 2, we demonstrate how the ionization state distribution in the SD-200 model strongly differs from the collisional ionization equilibrium (CIE, Mazzotta et al. 1998) on examples of the $\mathrm{O}^{2+}$ and $\mathrm{O}^{3+}$ ions producing bright FIR lines. It is seen that in the rapidly cooling plasma these ions are abundant at significantly lower temperatures (down to $300 \mathrm{~K}$ and below) than in the CIE because of very rapid plasma cooling by means of line emission.

In the same figure, we present the oxygen ion emission measure distribution over temperature $\mathrm{d} E_{\mathrm{O}} / \mathrm{d}\left(\ln T_{\mathrm{e}}\right)$ in the SD-200 model, defined by Eq. (3). This distribution together with the line emissivity dependence on temperature allows one to calculate the relative contributions to the total line flux from different temperature intervals (see Sect. 2.2).

\footnotetext{
3 We performed our own computations of the post-shock plasma recombination and discovered that our oxygen ion distribution over ionization stages is rather similar to that presented in the lower panel of Fig. 3 of the Sutherland \& Dopita (1995), but only if the ion spectroscopic symbols in that figure are increased by unity. Therefore, we assume that Fig. 3 of Sutherland \& Dopita (1995) has a misprint and the ion spectroscopic symbols should be read, e.g., "O VII" instead of "O VI", "O VI" instead of "O V", etc. From this, it follows that the SD-200 model values for the neutral oxygen abundances are not known and we do not provide estimates of the FIR [O I] line intensities.
} 


\subsection{Line flux computation}

The emissivity, $\mathrm{cm}^{3} / \mathrm{s}$, of a spectral line corresponding to transition from level $u$ to level $l$ is defined as

$\varepsilon(u, l)=n_{u} A_{u l} / n_{\mathrm{e}} n_{\mathrm{i}}$,

where $n_{\mathrm{e}}, n_{\mathrm{i}}$, and $n_{u}$ are the number densities of electrons, the corresponding ionic species, and ions in the state $u$, respectively, and $A_{u l}$ is the spontaneous $u \rightarrow l$ transition rate.

For oxygen ions at temperatures above $1000 \mathrm{~K}$, the line emissivities $\varepsilon$ were obtained from the Chianti atomic database (Dere et al. 1997; Landi et al. 2006). For lower temperatures and other ions, we computed emissivities using a standard approach, i.e., calculating the level populations by solving a system of linear equations describing transitions between the lowest ionic levels using electronic excitation effective collision strength data from the literature and spontaneous transition rates from both the Ralchenko et al. (2007) database ${ }^{4}$ and MCHF/MCDHF collection ${ }^{5}$, and then applying Eq. (1) to derive the line emissivities.

The electronic collisional excitation effective collision strength values were taken from Dufton \& Kingston (1991), Blum \& Pradhan (1992), Zhang et al. (1994), Butler \& Zeippen (1994), Lennon \& Burke (1994), Galavis et al. (1995), Pelan \& Berrington (1995), Berrington et al. (1998), Tayal \& Gupta (1999), Griffin \& Badnell (2000), Griffin et al. (2001), Tayal (2006), and Ramsbottom et al. (2007) and extrapolated to lower temperatures by a constant, if needed.

Line fluxes $I(u, l), \mathrm{erg} / \mathrm{cm}^{2} / \mathrm{s}$, were computed by integrating along the line of sight

$I(u, l)=h v \frac{S}{4 \pi R^{2}} \int \varepsilon\left(u, l ; T_{\mathrm{e}}(r)\right) n_{\mathrm{e}}(r) n_{\mathrm{i}}(r) \mathrm{d} r$,

where $h v$ is the photon energy, $R$ is the distance from the observer to the emitting region ( $3.4 \mathrm{kpc}$, Reed et al. 1995), and $S$ is the emitting region area. From this expression, it is seen that the line emission mostly originates in regions of the highest emission measure $n_{\mathrm{e}} n_{\mathrm{O}} \mathrm{d} r$, ionic abundance $n_{\mathrm{i}} / n_{\mathrm{O}}$, and emissivity $\varepsilon$ (here $n_{\mathrm{O}}$ is the total number density of all oxygen ions).

The integral over distance in the post-shock cooling region can be transformed into an integral over temperature by completing the substitution

$\mathrm{d} r=\frac{\mathrm{d} r}{\mathrm{~d} t} \frac{\mathrm{d} t}{\mathrm{~d} T_{\mathrm{e}}} \mathrm{d} T_{\mathrm{e}}, \quad \frac{\mathrm{d} r}{\mathrm{~d} t}=v_{\text {shock }} \frac{n_{0, \mathrm{t}}}{n_{\mathrm{t}}}$,

where $v_{\text {shock }}$ is the shock front speed, and $n_{\mathrm{t}}$ and $n_{0, \mathrm{t}}$ are the total number densities of all ions in the plasma at a given point and before the shock, respectively (i.e., in the SD-200 model $\left.n_{0, \mathrm{t}}=100 \mathrm{~cm}^{-3}\right)$. It is assumed here that the photon heating is insignificant compared to the energy losses.

The parameters of these equations, i.e., the cooling function, and electron and ion densities as functions of temperature - were taken from the SD-200 model.

The derivative $\mathrm{d} t / \mathrm{d} T_{\mathrm{e}}$ in the post-shock cooling region may be obtained from the energy conservation law written for one particle as

$\mathrm{d}\left(\frac{3}{2} \frac{n_{\mathrm{e}}+n_{\mathrm{t}}}{n_{\mathrm{t}}} k_{\mathrm{B}} T_{\mathrm{e}}\right)=-n_{\mathrm{e}} \Lambda_{\mathrm{N}} \mathrm{d} t-p \mathrm{~d}\left(\frac{1}{n_{\mathrm{t}}}\right)$,

where $k_{\mathrm{B}}$ is the Boltzmann constant, $\Lambda_{\mathrm{N}}$ is the cooling function (Sutherland \& Dopita 1993), and $p=\left(n_{\mathrm{e}}+n_{\mathrm{t}}\right) k_{\mathrm{B}} T_{\mathrm{e}}$ is the gas

\footnotetext{
${ }_{4}$ Available online at http://physics.nist.gov/asd3

5 http://atoms.vuse.vanderbilt.edu/
}

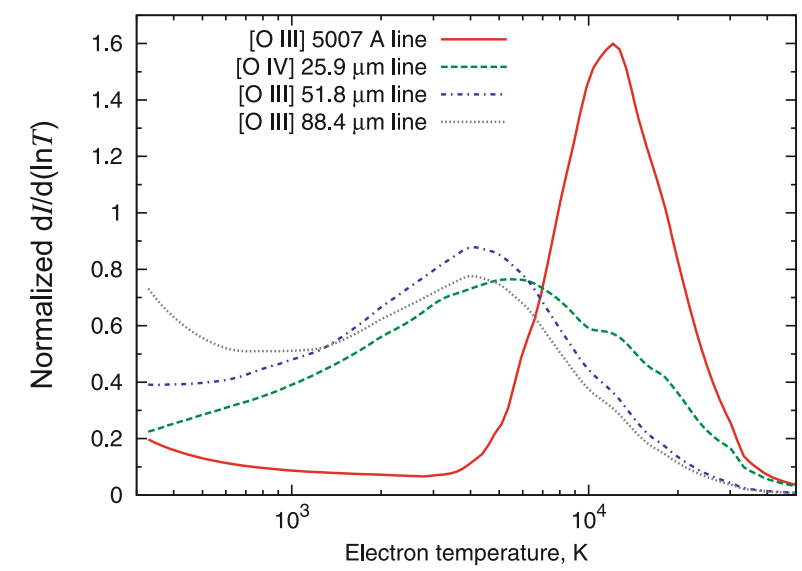

Fig. 3. Differential flux contribution per logarithmic temperature interval for the optical [O III] $5007 \AA$ line and infrared oxygen lines in the cooling post-shock region according to the SD-200 model. Curves are normalized so that the area enclosed under each of them equals one. Low-temperature (below $4000 \mathrm{~K}$ ) contribution to the optical [O III] line is due to recombination.

pressure. Dividing both sides by $\mathrm{d} T_{\mathrm{e}}$, we can now obtain the required derivative from the expression

$\frac{3}{2} k_{\mathrm{B}} T_{\mathrm{e}} \frac{\mathrm{d}\left(n_{\mathrm{e}} / n_{\mathrm{t}}\right)}{\mathrm{d} T_{\mathrm{e}}}+\frac{3}{2}\left(\frac{n_{\mathrm{e}}}{n_{\mathrm{t}}}+1\right) k_{\mathrm{B}}=-n_{\mathrm{e}} \Lambda_{\mathrm{N}} \frac{\mathrm{d} t}{\mathrm{~d} T_{\mathrm{e}}}+\left(\frac{n_{\mathrm{e}}}{n_{\mathrm{t}}}+1\right) \frac{k T_{\mathrm{e}}}{n_{\mathrm{t}}} \frac{\mathrm{d} n_{\mathrm{t}}}{\mathrm{d} T_{\mathrm{e}}}$.

We note that in the SD-200 model, the plasma pressure is not constant in the post-shock cooling region (their Fig. 3) at low temperatures because of the contribution of the magnetic field pressure.

For the purpose of qualitative analysis, we introduce the oxygen differential emission measure per logarithmic temperature interval

$\frac{\mathrm{d} E_{\mathrm{O}}}{\mathrm{d}\left(\ln T_{\mathrm{e}}\right)}=T_{\mathrm{e}} \frac{n_{\mathrm{O}} n_{\mathrm{e}} \mathrm{d} r}{\mathrm{~d} T_{\mathrm{e}}}$.

It indicates contribution of a given logarithmic temperature interval to the total emission measure, showing where most of the line emission originates. Using this notation, we can express the line flux given by Eq. (2) as

$I(u, l)=h v \frac{S}{4 \pi R^{2}} \int \varepsilon\left(u, l ; T_{\mathrm{e}}\right) \frac{\mathrm{d} E_{\mathrm{O}}}{\mathrm{d} \ln T_{\mathrm{e}}} \frac{n_{\mathrm{i}}}{n_{\mathrm{O}}} \frac{\mathrm{d} T_{\mathrm{e}}}{T_{\mathrm{e}}}$.

Contributions from different temperature intervals to the emission of the post-shock cooling region in the oxygen lines are illustrated in Fig. 3, where we plot the normalized line fluxes per logarithmic temperature interval. It is seen that the post-shock contribution to the visible [O III] line emission originates at temperatures of $(5-20) \times 10^{3} \mathrm{~K}$, whereas the infrared lines are emitted mostly at lower temperatures. A far broader range of temperatures also contributes to the line emission because of lower excitation energies.

In our analysis, we artificially stop integrating Eq. (2) in the post-shock region when the plasma temperature drops below $300 \mathrm{~K}$, since the SD-200 model does not contain data for lower temperatures. This should not influence the total FIR line intensities much, since the model predicts that they are mainly emitted in the pre-shock region.

In the pre-shock PIR, the ion density is constant and the temperature dependence on time for the SD-200 model is known explicitly. Therefore, Eq. (2) was applied directly to compute the line fluxes from this region. The resulting line flux ratios are given in Table 1. 


\section{Archival observations of the FIR lines}

The first search for the [OI] $63.19 \mu \mathrm{m}$ line was performed by Dinerstein et al. (1987), who observed the northern part of the Cas A using the NASA Kuiper Airborne Observatory. This search resulted in a 3- $\sigma$ upper limit to the reddening-corrected flux ratio $I(63.19 \mu \mathrm{m}) / I(5007 \AA)$ of about 0.3 , which tightly constrained some theoretical models. Later, the far-infrared lines of $\mathrm{O}, \mathrm{Ne}, \mathrm{Si}, \mathrm{S}$, and Ar ions were detected by the Infrared Space Observatory (ISO, Arendt et al. 1999). We describe our analysis of some of these archival observations in Sect. 3.1. The Spitzer Space Telescope recently spectrally mapped the Cas A supernova remnant (Ennis et al. 2006; Rho et al. 2008). In Sect. 3.2, we determine the line ratios $^{6}$ of $\mathrm{O}, \mathrm{Ne}, \mathrm{Si}, \mathrm{S}, \mathrm{Ar}$, and Fe lines to the [O III] $5007 \AA$ line.

To determine these ratios, we compare the infrared observations of ISO and Spitzer with the optical images of the Hubble Space Telescope. The most accurate way of comparing these observations would be an analysis of the properties of individual knots but, unfortunately, the ISO data are of insufficient angular resolution (about 40"). The angular resolution of the Spitzer data is superior $\left(2^{\prime \prime}-8^{\prime \prime}\right)$, but also too coarse to isolate the contributions of individual knots distinguishable within the optical maps $\left(0.2-1^{\prime \prime}\right.$, corresponding to $(3-15) \times 10^{16} \mathrm{~cm}$ at $\left.3.4 \mathrm{kpc}\right)$.

As the optical observations show, the bright FMK lifetime is of the order of 30 years (Kamper \& van den Bergh 1976), which is determined by the time needed by the shock to cross the cloud. After that, the knots fade, but others gradually appear in the maps of the Cas A. Therefore, one may directly compare either observations performed with a short time difference between them (as in the case of Hubble Space Telescope observations compared with Spitzer data), or averaged over a large area (as in the case of the ISO data).

\subsection{ISO observations}

The Infrared Space Observatory ${ }^{7}$ Long Wavelength Spectrometer (ISO LWS) observations of the Cassiopeia A supernova remnant were performed in 1996 and 1997. Here we do not discuss observations at shorter wavelengths $(\lambda<40 \mu \mathrm{m})$, since more recent data of significantly higher angular resolution is available from the Spitzer observatory.

To determine the line fluxes, we used the ISO LWS calibrated data from a highly-processed data product "uniformly processed LWS L01 spectra" available from the ISO data archive $^{8}$.

There are seven LWS observations in L01 mode each covering the full instrument spectral range between 43 and $170 \mu \mathrm{m}$ with medium spectral resolution $(\lambda / \Delta \lambda$ between 150 and 200$)$. The regions observed by ISO are shown in Fig. 4 overlaid on the Hubble Space Telescope Advanced Camera for Surveys (HST ACS) Cas A image in filter $F 475 W$, containing [O III] 4959 and $5007 \AA$ lines (Obs.ID 10286, observations completed in December 2004, Fesen et al. 2006).

\footnotetext{
6 Here and everywhere below the "line ratio" denotes the ratio of the line fluxes in energy units.

7 Results in this section are based on observations with ISO, an ESA project with instruments funded by ESA Member States (especially the PI countries: France, Germany, the Netherlands, and the United Kingdom) and with the participation of ISAS and NASA.

8 http://WwW.iso.vilspa.esa.es/ida/
}

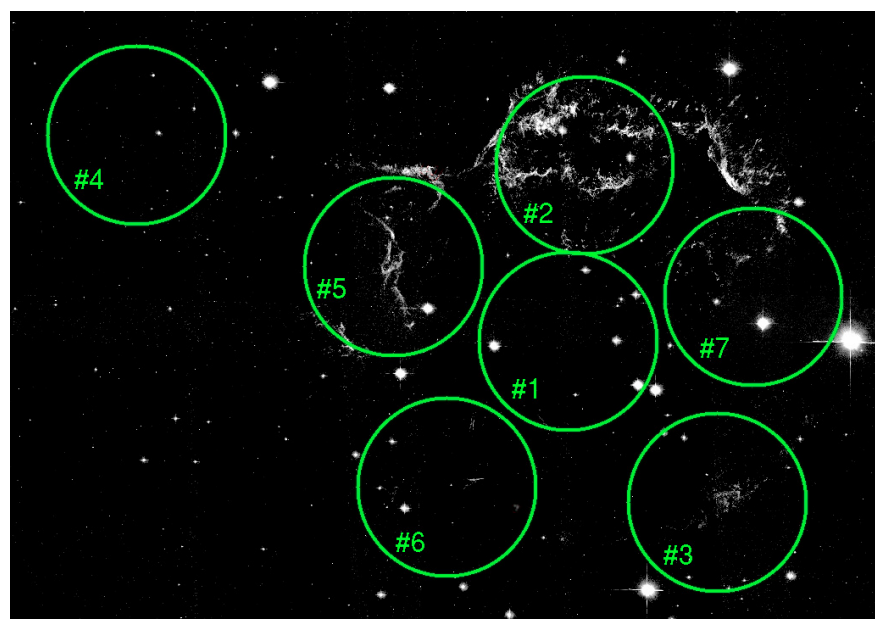

Fig. 4. ISO LWS apertures (green circles) overlaid on the Hubble Space Telescope 2004 ACS image of Cas A in $F 475 W$ filter (Fesen et al. 2006). North is upwards and East is to the left.

Table 2. Summary of the ISO LWS archival observations of the Cassiopeia A.

\begin{tabular}{lllll}
\hline \hline ID & Date & Exposure & RA & Dec \\
\hline Cas A \#1 & 1996 Jun. 24 & 1054 s & 350.8656 & +58.8130 \\
Cas A \#2 & 1996 Jun. 24 & $1052 \mathrm{~s}$ & 350.8614 & +58.8361 \\
Cas A \#3 & 1996 Jun. 24 & $1054 \mathrm{~s}$ & 350.8279 & +58.7919 \\
Cas A \#4 & 1996 Jun. 24 & $1054 \mathrm{~s}$ & 350.9748 & +58.8400 \\
Cas A \#5 & 1997 Jun. 09 & 1612 s & 350.9097 & +58.8228 \\
Cas A \#6 & 1997 Jun. 09 & 1612 s & 350.8963 & +58.7939 \\
Cas A \#7 & 1997 Jun. 02 & 1614 s & 350.8186 & +58.8188 \\
\hline
\end{tabular}

The ISO LWS observational data are summarized in Table 2 and the spectral cuts containing the discussed lines are presented in Fig. 5.

To compute the far-infrared spectral line fluxes, we subtracted the background continuum flux and averaged over three scans of the same detector, weighting the data according to its uncertainties. In the case of the [O III] $88.36 \mu \mathrm{m}$ line, it was possible to estimate the systematic uncertainties by comparing the flux values obtained from two neighboring detectors. The flux differences are expected to have two causes: slightly different apertures of the detectors and systematic errors due to imperfect instrumental calibration (Gry et al. 2003). Our analysis showed that differences between the two detector measurements do not exceed $10-15 \%$, when the line flux determination accuracy is sufficiently high (see Fig. 5 for a visual comparison).

The background-subtracted FIR line fluxes from the observed regions are presented in Table 3. It is seen that the flux at the level of up to $10 \%$ in the brightest fields is observed in the $88.36 \mu \mathrm{m}$ in the region \#4 situated outside the main supernova remnant shell with no detected optical [O III] emission and very little diffuse emission in other optical bands. We consider the flux from this region as originating in the foreground and/or background of Cassiopeia A and in our analysis subtract it from the fluxes of the other regions.

In the same table, we indicate the total fast-moving knot optical flux for the matched regions in the $F 475 \mathrm{~W}$ filter of the discussed HST ACS data. The diffuse emission in this filter is assumed to come only from the [O III] 4959 and $5007 \AA$ lines.

From these data, we calculated the line ratios to the [O III] $5007 \AA$ line, given in Table 4. Assuming that the FIR line emission observed by ISO originates in the same spatial regions as the 


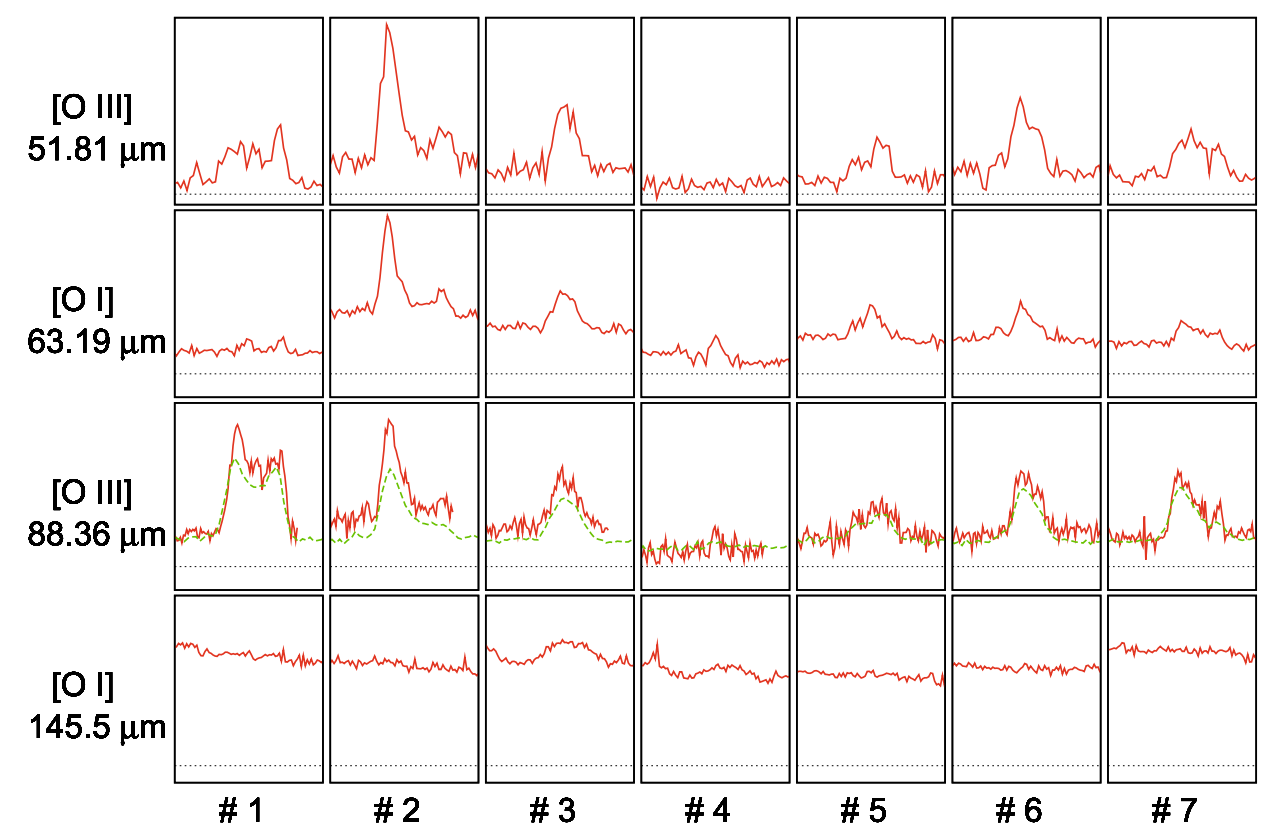

Fig. 5. ISO LWS spectra of Cas A at the observed locations (as shown in Fig. 4 and Table 2) are shown in the regions of oxygen far-infrared lines discussed in the text. Continuum emission is not subtracted. Plotted are the averages over three scans weighted according to their uncertainties. Two curves for the $88.36 \mu \mathrm{m}$ line correspond to measurements by two different ISO detectors. The short-dashed line denotes the zero level; maximum intensity corresponds to $1.7 \times 10^{-10}, 0.7 \times 10^{-10}, 0.7 \times 10^{-10}$, and $0.10 \times 10^{-10} \mathrm{erg} / \mathrm{cm}^{2} / \mu \mathrm{m}$ for [O III] $51.81 \mu \mathrm{m},[\mathrm{O}$ I] $63.19 \mu \mathrm{m},[\mathrm{O} \mathrm{III}] 88.36 \mu \mathrm{m}$, and $[\mathrm{OI}] 145.5 \mu \mathrm{m}$ lines, respectively. Spectral ranges are centered on the rest-frame wavelengths of the respective spectral lines and include a velocity range of $\pm 10^{4} \mathrm{~km} \mathrm{~s}^{-1}$.

Table 3. Far-infrared line fluxes from the ISO LWS observations of Cas A.

\begin{tabular}{llllllll}
\hline \hline Line & $\# 1$ & $\# 2$ & $\# 3$ & $\# 4$ & $\# 5$ & $\# 6$ & $\# 7$ \\
\hline \multicolumn{7}{c}{ FIR line fluxes $F, 10^{-11} \mathrm{erg} / \mathrm{cm}^{2} / \mathrm{s}$} \\
{$[\mathrm{O} \mathrm{III}] 52 \mu \mathrm{m}$} & 5.4 & 9.3 & 5.4 & $<1.2$ & 3.5 & 5.0 & 5.2 \\
{$[\mathrm{O} \mathrm{I}] 63 \mu \mathrm{m}$} & $<0.6$ & 2.6 & 1.2 & $<1.2$ & 1.0 & 1.0 & 0.9 \\
{$[\mathrm{O} \mathrm{III}] 88 \mu \mathrm{m}$} & 7.5 & 4.2 & 2.7 & 0.5 & 1.9 & 3.2 & 3.4 \\
{$[\mathrm{O}] 145 \mu \mathrm{m}$} & $<0.1$ & $<0.09$ & 0.3 & 0.1 & $<0.1$ & $<0.1$ & $<0.1$ \\
\hline$[\mathrm{O}$ III $]$ & $4959+5007 \AA ̊$ & line flux $F, 10^{-13} \mathrm{erg} / \mathrm{cm}^{2} / \mathrm{s}$ \\
& $<0.1$ & 25 & 2.0 & $<0.1$ & 6.5 & 0.5 & 2.9 \\
\hline
\end{tabular}

Fluxes from the matched regions in the HST ACS $F 475 W$ filter are also given. The upper limits are at the $3 \sigma$ level.

Note: ISO region positions are shown in Fig. 4. Regions \#3, \#6 and \#7 have higher interstellar absorption column (Bieging \& Crutcher 1986).

optical line emission, these flux ratios are characteristic of the knot emission and may be used for tests of the theoretical models. If significant large-scale diffuse emission in the FIR lines is present, as observed in the [S III] and [O IV] lines by Spitzer observations (see Sect. 3.2), then the determined line ratios should be interpreted as upper limits to the values characteristic of the knots.

In Table 4, we provide the values corrected for the interstellar absorption of the optical line using an average value from Hurford \& Fesen (1996). This level of reddening diminishes the observed visible [O III] doublet intensities by a factor of about $200\left(A_{V} \approx 5.0\right)$ and is characteristic of region \#2 and, possibly, region \#5 (Bieging \& Crutcher 1986).

For southern and western regions (\#3, \#6 and \#7) the reddening is known to be much higher because of absorption in the molecular clouds with peak $A_{V}$ 's reaching 10-15 (Bieging $\&$ Crutcher 1986) corresponding to the optical line attenuation of up to factors of $10^{4}$ and more. Therefore, we do not provide
Table 4. Far-infrared line flux ratios to the optical $5007 \AA$ line $I / I(5007 \AA)$ corrected for the optical line reddening.

\begin{tabular}{lll}
\hline \hline Line & $\# 2$ & $\# 5$ \\
\hline$[\mathrm{O}$ III $] 51.81 \mu \mathrm{m}$ & 0.25 & 0.36 \\
{$[\mathrm{O}$ I $63.19 \mu \mathrm{m}$} & 0.07 & 0.10 \\
{$[\mathrm{O}$ III $] 88.36 \mu \mathrm{m}$} & 0.10 & 0.14 \\
{$[\mathrm{O}$ I $] 145.5 \mu \mathrm{m}$} & $<0.0024$ & $<0.01$ \\
\hline
\end{tabular}

Note: the $5007 \AA$ Aline contribution of $75 \%$ to the total FMK emission in the $F 475 \mathrm{~W}$ filter is assumed. Optical line attenuation due to reddening of 200 times is assumed in both the ISO LWS regions, see text. FIR line attenuation is neglected.

dereddened line ratios in these regions. In our further analysis in Sects. 4 and 3.3, we use only the data from the region \#2.

There is an eight-year time difference between ISO and HST observations. During this time, which is about $30 \%$ of a bright FMK lifetime (Kamper \& van den Bergh 1976), some optical knots may have disappeared, and new ones may have appeared. However, averaging over a large number of optical knots contained in any ISO LWS field of view at least partially cancels changes induced by the brightening or fading of individual knots.

\subsection{Spitzer Space Telescope observations}

The Spitzer Space Telescope spectrally mapped the Cassiopeia $A$ in the infrared range using its Infrared Spectrograph (IRS) producing low-resolution spectra from 5 to $38 \mu \mathrm{m}$ (Ennis et al. 2006; Rho et al. 2008) and detected in this spectral band the fine-structure lines of [O IV], [Ne II], [Ne III], [Ne V], [Si II], [S III], [S IV], [Ar II], [Ar III], [Ar V], [Fe II], etc. 
We used basic calibrated data of these publicly available Spitzer observations (Program 3310) to construct the data cube over the entire remnant with the CUBISM software (Smith et al. 2007). The background regions were determined from the $8 \mu \mathrm{m}$ MIPS images of the supernova remnant (Hines et al. 2004).

The line flux maps were then produced using the parabolic approximation for the background continuum emission. This allowed the maps to be constructed reliably even for the weak lines in the regions of strong continuum dust emission.

Since the Spitzer spectral maps and the HST images were obtained almost simultaneously with a time difference of only one month, we were able to make a direct pixel-by-pixel comparison of the infrared line map with the optical image. For this purpose, we processed the HST images by removing the stars, convolving with the point spread function (PSF) of a given Spitzer spectral map computed as described in Appendix A, and regridding the HST image to match pixels to the IRS module containing each infrared line. These matched HST images were produced and compared to each of the infrared lines.

\subsubsection{The oxygen [O IV] $25.91 \mu \mathrm{m}$ line}

The [O IV] $25.91 \mu \mathrm{m}$ spectral line map of the northern Cas A shell region obtained from Spitzer observations is presented in Fig. 6. Alongside, we show the HST image in $F 475 \mathrm{~W}$ filter processed as described above. The northern region is of primary importance as it has a well-measured and almost constant interstellar absorption level.

It can be readily seen from the brightest features in Fig. 6 that there is a difference between the Cas A morphologies for the optical and infrared lines. The difference may have several reasons: variations in the interstellar reddening (Hurford \& Fesen 1996), emitting plasma chemical composition (Chevalier \& Kirshner 1979), and FMK pre-shock density distribution over the remnant.

In Fig. 7, we present the resulting pixel-by-pixel scatter plot showing interrelation between the [O IV] infrared and [O III] optical line fluxes. From this comparison of the maps, we derive the average line ratio of the optical and infrared lines, which corresponds to an average reddening, FMK density, and chemical composition.

In this analysis, "average" reddening was assumed to diminish the observed optical [O III] line emission by a factor of 200, which is an average of the Hurford \& Fesen (1996) measurements. As may be inferred from their paper, small-scale variations in the interstellar reddening in this region change the optical line attenuation within a factor of two. The observed scatter of the line ratio seen in Fig. 7 is of similar magnitude.

In the same figure, we also present data for five FMKs with reddening measured by Hurford \& Fesen (1996). Their positions are shown in Fig. 7. In these cases, the reddening correction was made according to their measurement results. Four out of five points lie very close to the best-fit lines, showing that a considerable part of the FMKs have similar excitation conditions. A remaining point (FMK 1) has relatively weaker [O IV] emission, showing that the physical conditions there are different. This region (Filament 1 of Baade \& Minkowski 1954) indeed has a composition that differs from that of the other regions (Chevalier \& Kirshner 1979).

In Fig. 6, infrared emission in the [OIV] line that originates in the central Cas A region with no optical nebulosity (see also Rho et al. 2008) is also noticeable. In Fig. 7, this infraredonly emission shows up on the left as horizontal branches, corresponding to an approximately constant background surface
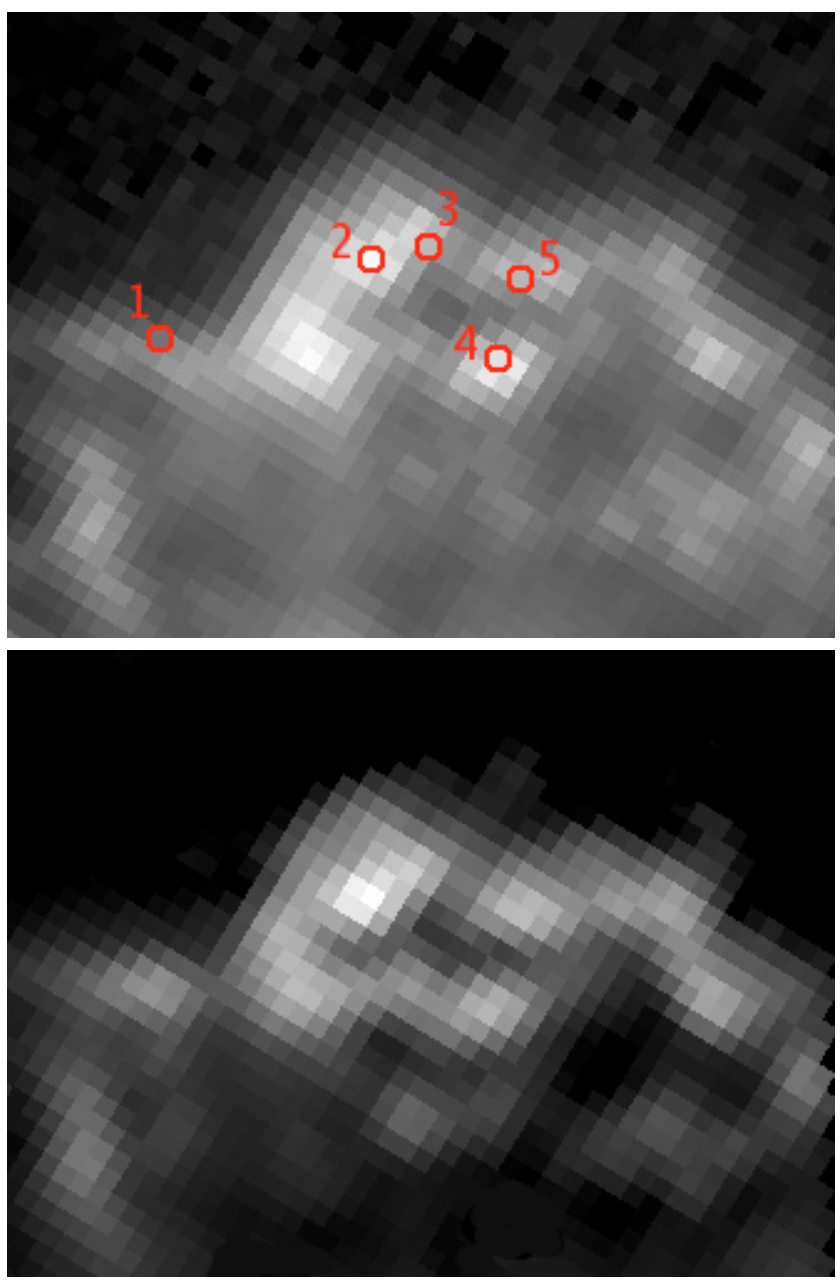

Fig. 6. The Spitzer Space Telescope map of the northern part of the Cas A optical shell rich in FMKs in the [OIV] line at $25.91 \mu \mathrm{m}$ (top) compared with the HST image in $F 475 W$ filter matched in resolution and in pixels (bottom). The maps are centered on RA of $350.8614 \mathrm{deg}$ and declination of $58.8361 \mathrm{deg}$ and show the region of $2.5 \times 3.5 \mathrm{arcmin}$. The red circles denote regions with reddening measured by Hurford \& Fesen (1996). Square root scale in intensity is used to enhance weak features. Diffuse infrared [O IV] emission from optically-dark regions is clearly visible.

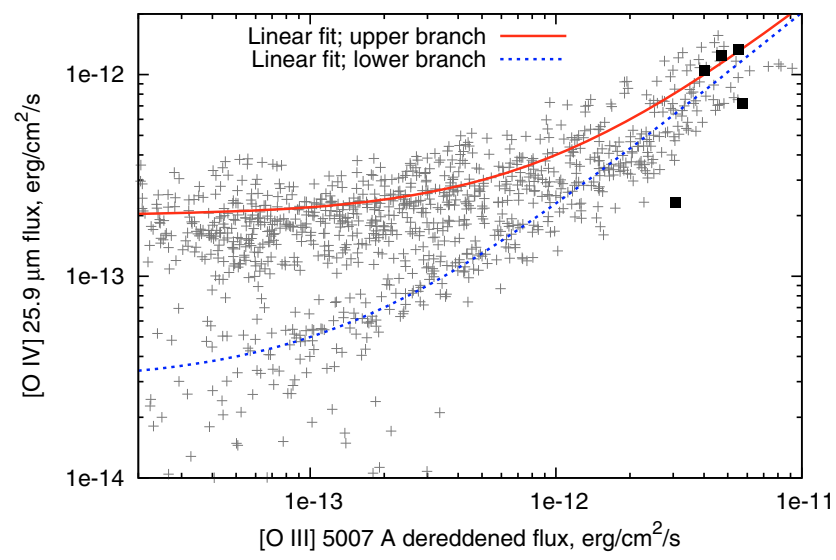

Fig. 7. Pixel-by-pixel comparison of the dereddened $5007 \AA$ line flux with the infrared [O IV] line map. Fluxes are given per detector pixel, equal to $\left(55^{\prime \prime} 08\right)^{2}$ for the IRS LL module containing the infrared oxygen line. Two linear fits, both with the same slope of 0.20 , are shown with lines. Filled squares denote FMKs from Hurford \& Fesen (1996) with measured reddening. 
Table 5. Infrared line fluxes $I$ and their ratios $I / I(5007 \AA)$ to the dereddened optical [O III] $5007 \AA$ A line from the Spitzer data.

\begin{tabular}{|c|c|c|c|c|c|c|c|c|c|c|c|}
\hline \multirow[t]{2}{*}{ Line } & \multicolumn{5}{|c|}{ Line flux $I, 10^{-13} \mathrm{erg} / \mathrm{cm}^{2} / \mathrm{s} /$ pixel } & \multicolumn{5}{|c|}{ Flux ratio $I / I(5007 \AA)$} & \multirow[b]{2}{*}{ FMK 5} \\
\hline & FMK 1 & FMK 2 & FMK 3 & FMK 4 & FMK 5 & Average & FMK 1 & FMK 2 & FMK 3 & FMK 4 & \\
\hline [Ar II] $6.985 \mu \mathrm{m}$ & 3.8 & 14 & 17 & 5.6 & 11 & $0.5(1)$ & 0.41 & 0.75 & 1.15 & 0.46 & 0.79 \\
\hline [Ar III] $8.991 \mu \mathrm{m}$ & 0.46 & 2.0 & 1.7 & 0.80 & 1.5 & $0.08(3)$ & 0.05 & 0.13 & 0.12 & 0.07 & 0.12 \\
\hline$[\mathrm{S} \mathrm{IV}] 10.51 \mu \mathrm{m}$ & 0.24 & 1.6 & 1.4 & 0.67 & 0.91 & $0.07(2)$ & 0.025 & 0.11 & 0.10 & 0.06 & 0.07 \\
\hline$[\mathrm{Ne}$ II $] 12.81 \mu \mathrm{m}$ & 0.31 & 0.92 & 0.89 & 1.0 & 0.30 & $0.10(8)$ & 0.04 & 0.07 & 0.07 & 0.08 & 0.025 \\
\hline$[\mathrm{Ne} \mathrm{V}] 14.32 \mu \mathrm{m}$ & 0.071 & 0.20 & 0.18 & 0.10 & 0.14 & $0.012(7)$ & 0.009 & 0.012 & 0.012 & 0.009 & 0.014 \\
\hline$[\mathrm{Ne}$ III] $15.56 \mu \mathrm{m}$ & 0.29 & 2.2 & 1.2 & 3.0 & 0.47 & $0.05(3)$ & 0.009 & 0.03 & 0.03 & 0.05 & 0.006 \\
\hline$[\mathrm{Fe}$ II $] 17.94 \mu \mathrm{m}$ & 0.16 & 0.90 & 1.3 & 1.8 & 0.87 & $0.03(2)$ & 0.005 & 0.015 & 0.03 & 0.04 & 0.013 \\
\hline [S III] $18.71 \mu \mathrm{m}$ & 0.82 & 5.0 & 5.2 & 2.5 & 4.2 & $0.08(2)$ & 0.025 & 0.08 & 0.15 & 0.05 & 0.06 \\
\hline [O IV $] 25.91 \mu \mathrm{m}$ & 2.3 & 13 & 11 & 12 & 7.2 & $0.20(5)$ & 0.08 & 0.24 & 0.26 & 0.26 & 0.13 \\
\hline [S III] $33.48 \mu \mathrm{m}$ & 0.28 & 1.9 & 2.3 & 0.76 & 0.6 & $0.04(1)$ & 0.011 & 0.05 & 0.06 & 0.015 & 0.015 \\
\hline [Si II] $34.81 \mu \mathrm{m}$ & 1.0 & 3.7 & 3.9 & 1.7 & 1.7 & $0.07(3)$ & 0.04 & 0.10 & 0.11 & 0.03 & 0.04 \\
\hline
\end{tabular}

The column "average" contains the ratio values averaged over the northern part of Cas A and their observed scatter, and other columns contain data for the Hurford \& Fesen (1996) FMKs.

Notes: the table contains data for the strongest lines only. The regions are numbered as in Hurford \& Fesen (1996) with their positions shown in Fig. 7. The "average" column assumes single optical line reddening correction of 200 times. Note that the pixel sizes are different for the IRS LL $\left(5{ }^{\prime \prime} 08\right.$ or $\left.6.06 \times 10^{-10} \mathrm{sr}, \lambda>15 \mu \mathrm{m}\right)$ and SL $\left(1^{\prime \prime} 85\right.$ or $\left.8.04 \times 10^{-11} \mathrm{sr}, \lambda<15 \mu \mathrm{m}\right)$ modules.

brightness. Two of these branches are apparent, probably corresponding to two different column densities of the $\mathrm{O}^{3+}$ ion. Pixels forming the lower branch are situated just outside the optical shell, where the optical surface brightness is already fading, but the infrared line emission remains present.

The upper branch corresponds to the diffuse emission in the central part of the supernova remnant. Corresponding points in the HST image are partially outside the Fig. 7 plot range, but the infrared line surface brightness remains on the level of $(1-3) \times$ $10^{-13} \mathrm{erg} / \mathrm{cm}^{2} / \mathrm{s} / \mathrm{pix}$.

This infrared-only line emission is consistent with the existence of extended cold photoionized regions invisible in optical and X-ray emission close to the center of the supernova remnant, possibly connected with the thermal radio absorption observed in the same regions (Kassim et al. 1995; Delaney 2004).

We note that the [O IV] infrared line at $25.91 \mu \mathrm{m}$ overlaps with the [Fe II] line at $25.99 \mu \mathrm{m}$, but the contribution of the latter can be estimated by comparison with the [Fe II] $17.94 \mu \mathrm{m}$ line map. We assume that the [Fe II] 25.99/17.94 $\mu$ m line ratio is constant over the remnant near the reverse shock. Then, we select a bright region of Cas A with a low value of the line flux ratio

$$
\frac{I([\mathrm{O} I V] 25.91 \mu \mathrm{m}+[\mathrm{Fe} \mathrm{II}] 25.99 \mu \mathrm{m})}{I([\mathrm{Fe} \mathrm{II}] 17.94 \mu \mathrm{m})} \approx 5,
$$

and assume it to be the upper bound to the [Fe II] 25.99/17.94 $\mu \mathrm{m}$ line ratio later used in Sect. 5.

This bound is used to infer that the upper limit to the $25.99 \mu \mathrm{m}$ [Fe II] line contribution to the total flux of the observed line near $26 \mu \mathrm{m}$ is about $20-40 \%$ in all regions of the Cas A used in our analysis (i.e., the Hurford \& Fesen (1996) FMKs).

\subsubsection{Other infrared lines in the Spitzer spectral maps}

Similar pixel-by-pixel comparisons with the optical image were also performed for other bright spectral line flux maps. In Table 5, we provide the derived flux ratios to the optical [O III] $5007 \AA$ line in five Hurford \& Fesen (1996) FMKs and the average values of these ratios in the northern part of the Cas A shell. The last digit in brackets in the "average" column denote the observed 2- $\sigma$ scatter around the average line flux ratio in units of the last digit. This scatter represents the true variations in the line flux ratios due to both chemical composition variations (Chevalier \& Kirshner 1979) and variations in physical excitation conditions (e.g., different pre-shock density in different regions of $\mathrm{Cas} \mathrm{A})$.

From the table, it can be seen that different line flux ratios have different scatter around their average values. A small scatter in e.g., the Ar, Si, and S line ratios to the $5007 \AA$ line implies that the Cas A morphology in these lines is similar to the optical one. A larger scatter, present in e.g., $\mathrm{Ne}$ and $\mathrm{Fe}$ line ratios to the $5007 \AA$ line corresponds to different morphologies of the infrared spectral maps, as also noted by Rho et al. (2008).

The obtained line ratios may also be used as tests of the theoretical models and are utilized in the next section to estimate the average abundances and physical conditions in the bright Cas $\mathrm{A}$ knots.

From Table 5, it is seen that the full energy flux in the infrared lines detected by Spitzer exceeds one of the brightest optical line - [O III] $5007 \AA$ - and is of the same order as the total power in all optical lines summed up.

\subsection{Comparison of the model predictions with observations}

As the models provide predictions only for the oxygen line relative intensities, we cannot use observations of lines of other ions to directly compare with the models.

Comparing observed far-infrared line ratios with theoretical model results (see Table 1), we see that the Itoh (1981a,b) models underestimate the [O III] and [O IV] line fluxes by factors of $3-40$, as a result of having a far lower average ionization degree after the shock passage than in other models. In contrast, the Borkowski \& Shull (1990) model BS-F and the Sutherland \& Dopita (1995) model SD-200 that do not account for electron conductivity, both overpredict the emission in the [O IV] line by factors of 20-40.

The remaining model (BS-DC of Borkowski \& Shull 1990) predicts the infrared line fluxes to within a factor of several, except for the neutral oxygen lines, but this problem is at least partially resolved by increasing the pre-shock density (see Sect. 4.1.2). This strongly suggests that taking into account the electron conductivity is essential for a model when attempting to reproduce the observations. 
D. Docenko and R. A. Sunyaev: Fine-structure infrared lines from the Cassiopeia A knots

Table 6. Information from the same ion line flux ratios measured in the FMKs. (See text for details).

\begin{tabular}{|c|c|c|c|c|c|c|c|c|c|}
\hline \multirow[b]{2}{*}{ spectrum } & \multirow[b]{2}{*}{ line $a$} & \multirow[b]{2}{*}{ line $b$} & \multirow{2}{*}{$\begin{array}{l}\text { Flux } \\
\text { ratio }\end{array}$} & \multicolumn{2}{|c|}{ Single region } & \multicolumn{4}{|c|}{ Two regions } \\
\hline & & & & $T_{\mathrm{e}}, \mathrm{K}^{*}$ & $n_{\mathrm{e}}, \mathrm{cm}^{-3}$ & $R_{1}$ & $R_{2}$ & $f_{\mathrm{PIR}, a}$ & $f_{\mathrm{PIR}, b}$ \\
\hline$\overline{[\mathrm{O} I]}$ & $63.19 \mu \mathrm{m}$ & $145.5 \mu \mathrm{m}$ & $>30$ & $\begin{array}{l}<100 \\
200-1 \times 10^{4}\end{array}$ & $\begin{array}{l}\text { any } \\
>2 \times 10^{5}\end{array}$ & & & & \\
\hline [O III] & $51.81 \mu \mathrm{m}$ & $88.36 \mu \mathrm{m}$ & 2.5 & $300-1.5 \times 10^{4}$ & $500-1000$ & 1.1 & 9.7 & 0.40 & 0.85 \\
\hline [O III] & $88.36 \mu \mathrm{m}$ & $5007 \AA$ & 0.10 & $\begin{array}{l}1.0 \times 10^{4} \\
1.5 \times 10^{4}\end{array}$ & $\begin{array}{l}800 \\
200\end{array}$ & & & $\begin{array}{l}1.0^{*} \\
1.0^{*}\end{array}$ & $\begin{array}{l}1.0^{*} \\
1.0^{*}\end{array}$ \\
\hline $\begin{array}{l}{[\mathrm{Ne} \text { III] }} \\
{[\mathrm{Ne} \text { }]}\end{array}$ & $\begin{array}{l}36.01 \mu \mathrm{m} \\
24.32 \mu \mathrm{m}\end{array}$ & $\begin{array}{l}15.56 \mu \mathrm{m} \\
14.32 \mu \mathrm{m}\end{array}$ & $\begin{array}{l}<0.08 \\
<0.2\end{array}$ & $\begin{array}{l}\text { any } \\
<3 \times 10^{4}\end{array}$ & $\begin{array}{l}\text { any } \\
>3 \times 10^{4}\end{array}$ & & & & \\
\hline $\begin{array}{l}\text { [S III] } \\
{[\mathrm{Ar} \text { III] }}\end{array}$ & $\begin{array}{l}33.48 \mu \mathrm{m} \\
21.83 \mu \mathrm{m}\end{array}$ & $\begin{array}{l}18.71 \mu \mathrm{m} \\
8.991 \mu \mathrm{m}\end{array}$ & $\begin{array}{l}0.5 \\
<0.10\end{array}$ & $\begin{array}{l}600-3 \times 10^{4} \\
\text { any }\end{array}$ & $\begin{array}{l}1500-3000 \\
\text { any }\end{array}$ & 1.8 & 0.09 & 0.85 & 0.25 \\
\hline [Ar V] & $7.914 \mu \mathrm{m}$ & $13.07 \mu \mathrm{m}$ & 1.6 & $1500-3 \times 10^{4}$ & $(4-10) \times 10^{4}$ & 1.0 & 6.7 & 0.5 & 0.9 \\
\hline$[\mathrm{Fe} \mathrm{II}]^{* * *}$ & $1.3209 \mu \mathrm{m}$ & $1.2946 \mu \mathrm{m}$ & 0.75 & any & $>2 \times 10^{5}$ & & & & \\
\hline$[\mathrm{Fe} \mathrm{II}]^{* * *}$ & $1.3209 \mu \mathrm{m}$ & $17.94 \mu \mathrm{m}$ & 0.024 & $\begin{array}{l}1700-3000^{* *} \\
1700-1800^{* *}\end{array}$ & $\begin{array}{l}\text { any } \\
>1 \times 10^{5 *}\end{array}$ & & & & \\
\hline
\end{tabular}

${ }^{*}$ Assumed value; ${ }^{* *}$ derived value; ${ }^{* * *}$ the $[\mathrm{Fe}$ II] line flux ratios are discussed in Sect. 5.1.

The [O III] far-infrared line ratio $I(51.81 \mu \mathrm{m}) / I(88.36 \mu \mathrm{m})$ in the Borkowski \& Shull (1990) models is significantly higher than observed (6 versus 2.5). This discrepancy arises because they do not include emission from the pre-shock photoionized region. In the SD-200 model, in contrast, the pre-shock region dominates the $[\mathrm{O}$ III] infrared line emission and diminishes this infrared line flux ratio to 1.2. Therefore, to reproduce the observed line ratio the pre-shock and post-shock contributions should be comparable (see also Table 6).

The model I-H provides the most accurate description of the neutral oxygen FIR line ratio. In this model, most of the [O I] emission originates in the post-shock PIR. The line ratio corresponds to the high-density limit, but the predicted line intensities are about 20 times stronger than observed. As mentioned in Sect. 4.1.2, this discrepancy may be reduced if we assume higher pre-shock atom number density than in the model (for example, $300 \mathrm{~cm}^{-3}$ instead of $30 \mathrm{~cm}^{-3}$ ), as the line emissivities decrease, but both the ionization parameter and the column density of the PIR remain the same.

\subsection{Energetics of the infrared emission}

From the analysis of the Spitzer archival observations, it is straightforward to determine that in the wavelength range from 5 to $35 \mu \mathrm{m}$ the total Cas A luminosity is $3.3 \times 10^{37} \mathrm{erg} / \mathrm{s}$ (the luminosity values are computed assuming a distance to Cas A of $3.4 \mathrm{kpc}$; Reed et al. 1995). The total spectral line luminosity in the same range is found to be one order of magnitude lower, contributing $3.0 \times 10^{36} \mathrm{erg} / \mathrm{s}$.

For the longer wavelengths between $35 \mu \mathrm{m}$ and $1 \mathrm{~mm}$, we estimated the total Cas A luminosity using data published by Hines et al. (2004) to be about $1.5 \times 10^{37} \mathrm{erg} / \mathrm{s}$. The line contribution to this value in the narrower ISO LWS range between 40 and $200 \mu \mathrm{m}$ is about $9 \times 10^{35} \mathrm{erg} / \mathrm{s}$, as follows from Table 3 . Therefore, the total Cas A dust continuum and line luminosities in the infrared range between $5 \mu \mathrm{m}$ and $1 \mathrm{~mm}$ are $4.7 \times 10^{37}$ and $3.9 \times 10^{36} \mathrm{erg} / \mathrm{s}$, respectively.

It is interesting to compare these values with the X-ray and radio luminosities. In radio, the total luminosity at wavelengths longer than $1 \mathrm{~mm}$ is about $1.0 \times 10^{35} \mathrm{erg} / \mathrm{s}$ as calculated directly from the spectrum provided by Baars et al. (1977) and Hines et al. (2004), i.e., much lower than in the infrared.

It is not so straightforward to determine the total Cas A luminosity in X-rays because of interstellar and internal X-ray absorption. Various estimates result in values corrected for the absorption of $(1-5) \times 10^{37} \mathrm{erg} / \mathrm{s}$ (Hartmann et al. 1997; Zombeck 2007), but are dependent on the assumed spectral model and absorbing material column density.

It can be seen from this comparison that the infrared continuum emission is as an important radiative energy loss mechanism from the supernova remnant as the X-rays.

It is easy to notice that the infrared line-to-continuum emission ratio in the Spitzer range is variable over the supernova remnant, as indicated also by the spectra provided by Rho et al. (2008), with a typical value of 0.10 . The highest values of this ratio of about 0.22 are characteristic of the central Cas A region. In these regions, the energy losses in the line emission are relatively twice as high as average, although, still significantly below the losses in the infrared continuum.

\section{Physical conditions and abundances in the FMKs}

The measured line fluxes may be used directly to determine the ionic abundances ${ }^{9}$, if the model of the emitting region (temperature and density distribution) is known. Sicne the models do not reproduce observations precisely enough (see Table 1), we cannot assume that this distribution is known for the FMKs.

Thus, the line fluxes cannot be used in such a direct fashion and the inferred ionic abundances will depend on the assumed physical conditions. Fortunately, emissivities of some of the finestructure lines are only weakly dependent on the electron densities and temperatures accross a rather wide range, allowing us to use these lines for the abundance determination even without detailed knowledge of the line-emitting region properties.

An analysis of the measured flux ratios of lines of the same ion is a more powerful method. These line ratios may be used directly to constrain the temperature and density of the emitting region, provided that the emitting region is uniform and physically the same for both lines defining the ratio.

However, these assumptions are not fulfilled in the fastmoving knots of most line pairs and we cannot obtain emitting region parameters from, e.g., a comparison of optical and infrared lines of [O III]. Even the density-sensitive ratios of two infrared lines should be interpreted carefully, as from the theoretical models it follows that their emission may originate both in the pre-shock and post-shock regions that have comparable

\footnotetext{
9 In this paper, by abundance we denote a ratio of number density of given ions or atoms to the number density of all oxygen ions (i.e., $n / n_{\mathrm{O}}$ ).
} 
emission measures of the order of $10^{19} \mathrm{~cm}^{-5}$ and temperatures of the order of $(0.5-2) \times 10^{4} \mathrm{~K}$, but densities that differ by a factor of a thousand or more.

More specifically, the pre-shock photoionized region has a low electron density (of the order of $100-250 \mathrm{~cm}^{-3}$ according to the SD-200 model) and produces lines with approximately low-density limit line ratio, and the post-shock cooling region $\left(n_{\mathrm{e}} \approx 10^{6} \mathrm{~cm}^{-3}\right)$ produces lines having high-density limit line ratio (i.e., the corresponding level populations are determined by collisional processes). Therefore, instead of determining the density, some of the FIR line ratios determine the contributions of the high- and low-density regions to the total line emission.

In the following subsections, we analyze the line flux ratios to infer the physical conditions and the line fluxes to estimate the ionic and elemental abundances.

\subsection{Information obtained from the same ion line flux ratios}

In the infrared range probed by the Spitzer and the ISO observatories, there are seven line pairs that can be used for plasma diagnostics, and have at least one of lines defining the pair detected. These line pairs correspond to transitions between three of the lowest fine structure states ${ }^{3} P_{0,1,2}$ in the ground $p^{2}$ and $p^{4}$ electron configurations. Of these seven diagnostic line pairs, three have both lines detected ([O III], [S III], and [Ar V] lines) and four have detections of just one of the lines ([O I], [Ne III], $[\mathrm{Ne} \mathrm{V}]$, and [Ar III] lines). The measured values of the flux ratios and the inferred physical parameters are summarized in Table 6.

\subsection{1. [O III] FIR line flux ratio}

The measured ratios of the far-infrared [O III] lines $I(51.81 \mu \mathrm{m}) / I(88.36 \mu \mathrm{m})$ in different ISO spectra lie between 0.7 and 2.5 (see Table 3) with some indication of a correlation between the line ratio and the optical line intensity (a correlation is also observed for the [S III] line ratio map compiled from the Spitzer data).

In the approximation of two emitting regions (pre-shock and post-shock), we use the following expression to determine the pre-shock PIR contribution to the total line $b$ flux from the measured $a$ and $b$ line flux ratio $R=I_{a} / I_{b}$ (see Appendix B for its derivation):

$f_{\mathrm{PIR}, b} \equiv \frac{I_{\mathrm{PIR}, b}}{I_{\mathrm{tot}, b}}=\frac{R_{2}-R}{R_{2}-R_{1}}$,

where $I_{\mathrm{PIR}, b}$ and $I_{\mathrm{tot}, b}$ denote the photoionized region and total line $b$ fluxes, respectively, and $R_{1}$ and $R_{2}$ denote theoretical line ratio values in the pre- and post-shock regions.

The [O III] line ratio of 2.5 observed for the ISO LWS region \#2 with many optical knots may thus be achieved only if $85 \%$ of the $88.36 \mu \mathrm{m}$ line and $40 \%$ of the $51.81 \mu \mathrm{m}$ line originate in the pre-shock region. The corresponding theoretical line ratios in this case are $R_{1}=1.1$ and $R_{2}=9.7$ (see Table 6).

As shown by Sutherland \& Dopita (1995), the $5007 \AA$ line mostly originates in the pre-shock region. We determined that this is also the case for the $88.36 \mu \mathrm{m}$ line. This allows us to take one further step and use their flux ratio of $I(88.36 \mu \mathrm{m}) / I(5007 \AA) \approx 0.10$ to estimate the pre-shock physical conditions in the region emitting these [O III] lines.

This line ratio is sensitive to both density and temperature and in a single-temperature emission region model corresponds

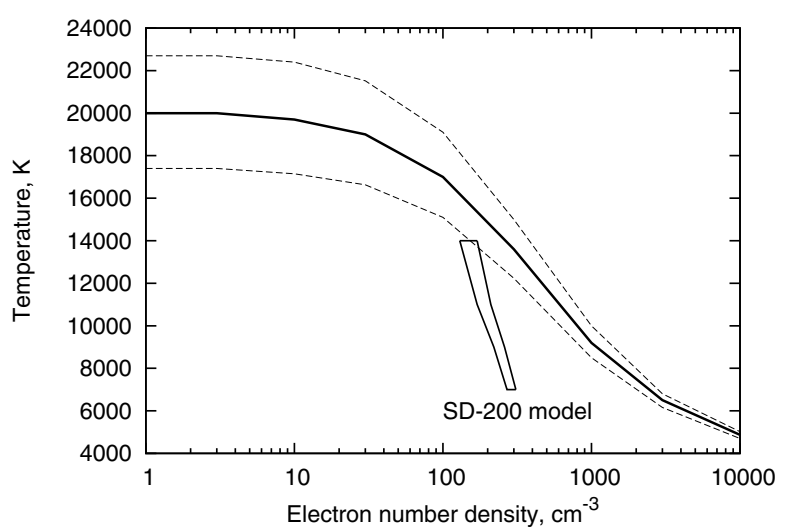

Fig. 8. The constraints on the pre-shock region emitting the [O III] lines from the average $I(88.36 \mu \mathrm{m}) / I(5007 \AA)$ line flux ratio (solid line). The dashed lines correspond to the constraints if the line flux ratio is changed by $20 \%$. The contour denotes the temperature-density relation in the region of the SD-200 model most intensely emitting [O III] lines.

to $n_{\mathrm{e}}$ of several hundreds $\mathrm{cm}^{-3}$ for temperatures $(1.0-1.5) \times$ $10^{4} \mathrm{~K}$ (see Table 6). These values are very similar to, but somewhat different from, ones given by the SD-200 model for which $T_{\mathrm{e}} \leq 1.1 \times 10^{4} \mathrm{~K}$ and $n_{\mathrm{e}} \approx 200 \mathrm{~cm}^{-3}$ in the region that most intensely emits both [O III] lines. The resulting observational constraint on the pre-shock line emission region is indicated in Fig. 8.

\subsection{2. [O I] FIR line flux ratio}

It is more difficult to use the [OI] line ratio for the quantitative analysis, because only one of the lines is detected. From ISO observations of region $\# 2$, we obtain the $3 \sigma$ lower limit to the $I(63.19 \mu \mathrm{m}) / I(145.5 \mu \mathrm{m})$ line ratio of about 30 .

Some information may still be obtained about this lower limit: it corresponds to either extremely low temperatures below $100 \mathrm{~K}$ or high electron densities above $10^{5} \mathrm{~cm}^{-3}$ (see Table 6).

According to the theoretical models, there is essentially no neutral oxygen in the post-shock cooling region because of slow recombination processes. The models also predict the rapid ionization of neutral oxygen in the pre-shock PIR with a very low line ratio $I(63.19 \mu \mathrm{m}) / I(5007 \AA)$ much less than 0.01 (the observed value is 0.07 , see Table 4$)$. The pre-shock region in which the $[\mathrm{O} \mathrm{I}]$ line originates, is also excluded from the observed value of the $I(63.19 \mu \mathrm{m}) / I(145.5 \mu \mathrm{m})$ line ratio.

From this, we conclude that the far-infrared [O I] line emission in the FMKs originates in the dense photoionized region after the shock.

All the models predict values of this line flux ratio with respect to the $5007 \AA$ line to be much higher than observed (see Table 1). Part of this discrepancy may be explained by the low model values for the pre-shock oxygen densities. For example, the Borkowski \& Shull (1990) models assume a very low preshock oxygen density of $1 \mathrm{~cm}^{-3}$. Taking it higher by two orders of magnitude will decrease the post-shock $63.19 \mu \mathrm{m}$ line intensity significantly. However, this is still not enough to reconcile the model predictions with the observations. The most probable reason for the low observed $I(63.19 \mu \mathrm{m}) / I(5007 \AA)$ line ratio is a combination of the post-shock region truncation and high preshock density, as discussed below in Sect. 5.5.

It should be noted that based on ISO data we cannot exclude part of the $63.19 \mu \mathrm{m}$ line originating in a cold $(T<100 \mathrm{~K}$ or so) diffuse medium outside the optical knots (although, see 
D. Docenko and R. A. Sunyaev: Fine-structure infrared lines from the Cassiopeia A knots

Table 7. Estimated FMK post-shock cooling region ionic abundances $n_{\text {ion }} / n_{\mathrm{O}}$. (See text for details).

\begin{tabular}{lllllll}
\hline \hline Line & $I / I(5007 \AA)$ & $T_{\mathrm{e}}, K$ & $\varepsilon, \mathrm{cm}^{3} / \mathrm{s}$ & $\Delta t, 10^{3} \mathrm{~s}$ & $n_{\text {ion }} / n_{\mathrm{O}}$ & $n_{\text {ion }} / n_{\mathrm{O}}(\mathrm{model})^{*}$ \\
\hline$[\mathrm{Si} \mathrm{X}] 1.430 \mu \mathrm{m}$ & 0.0016 & $2 \times 10^{4}$ & $2.9 \times 10^{-8}$ & 40 & $7 \times 10^{-5}$ & $5 \times 10^{-4}$ \\
{$[\mathrm{Si} \mathrm{VI}] 1.964 \mu \mathrm{m}$} & 0.004 & $1 \times 10^{4}$ & $3.1 \times 10^{-9}$ & 40 & 0.002 & 0.01 \\
{$[\mathrm{Mg} \mathrm{V}] 5.608 \mu \mathrm{m}$} & $<0.003$ & $6 \times 10^{3}$ & $9.0 \times 10^{-9}$ & 30 & $<0.002$ & $4 \times 10^{-4}$ \\
{$[\mathrm{Ar} \mathrm{V}] 7.914 \mu \mathrm{m}$} & $0.005^{* *}$ & $1 \times 10^{4}$ & $1.3 \times 10^{-8}$ & 60 & $8 \times 10^{-4}$ & $5 \times 10^{-5}$ \\
{$[\mathrm{Ne} \mathrm{V}] 14.32 \mu \mathrm{m}$} & 0.015 & $1 \times 10^{4}$ & $2.4 \times 10^{-9}$ & 50 & 0.06 & 0.002 \\
\hline
\end{tabular}

* Estimated based on SD-200 model. The elemental abundances assumed to deduce the model ionic abundances with respect to oxygen are given in Table $9 ;{ }^{* *}$ this line is partly arising in the pre-shock region. As follows from Table 6 , the post-shock region contribution is $I / I(5007 \AA) \approx 0.0025$.

Table 8. Estimated FMK pre-shock ionic abundances $n_{\text {ion }} / n_{\mathrm{O}}$ computed assuming that the lines originate only in the pre-shock PIR. (See text for details).

\begin{tabular}{lllllllll}
\hline \hline Line & $I / I(5007 \AA)$ & $T_{\mathrm{e}}, \mathrm{K}$ & $n_{\mathrm{e}}, \mathrm{cm}^{-3}$ & $\varepsilon, \mathrm{cm}^{3} / \mathrm{s}$ & $\mathrm{IP}, \mathrm{eV}$ & $\Delta t, \mathrm{~s}$ & $n_{\text {ion }} / n(\mathrm{O})$ & $n_{\text {element }} / n(\mathrm{O})^{* *}$ \\
\hline$[\mathrm{Ar} \mathrm{II}] 6.985 \mu \mathrm{m}$ & 0.5 & $2.0 \times 10^{4}$ & 100 & $4 \times 10^{-8}$ & 27.6 & $5 \times 10^{6}$ & $1.0^{*}$ & 0.005 \\
{$[\mathrm{Ar} \mathrm{V}] 13.07 \mu \mathrm{m}$} & 0.003 & 7000 & 250 & $8 \times 10^{-7}$ & 75.1 & $5 \times 10^{7}$ & $8 \times 10^{-5}$ & 0.005 \\
{$[\mathrm{Ne}$ II] $12.81 \mu \mathrm{m}$} & 0.10 & $1.5 \times 10^{4}$ & 150 & $5 \times 10^{-8}$ & 41.0 & $1.4 \times 10^{7}$ & 0.06 & 0.02 \\
{$[\mathrm{Fe} \mathrm{II}] 17.94 \mu \mathrm{m}$} & 0.03 & $2.0 \times 10^{4}$ & 50 & $1.1 \times 10^{-8}$ & 16.2 & $9 \times 10^{5}$ & $5.5^{*}$ & 0.005 \\
{$[\mathrm{~S} \mathrm{III}] 33.48 \mu \mathrm{m}$} & 0.04 & $1.5 \times 10^{4}$ & 150 & $3.0 \times 10^{-7}$ & 34.8 & $1 \times 10^{7}$ & 0.015 & 0.05 \\
{$[\mathrm{Si} \mathrm{II}] 34.81 \mu \mathrm{m}$} & 0.07 & $2.0 \times 10^{4}$ & 50 & $1.5 \times 10^{-7}$ & 16.4 & $1 \times 10^{6}$ & $0.7^{*}$ & 0.05 \\
{$[\mathrm{O} \mathrm{III}] 88.36 \mu \mathrm{m}$} & 0.10 & $1.0 \times 10^{4}$ & 200 & $4.0 \times 10^{-8}$ & 54.9 & $3 \times 10^{7}$ & 1.1 & 1.0 \\
\hline
\end{tabular}

${ }^{*}$ Shown to arises mostly in the post-shock photoionized region; see Sect. $5 ;{ }^{* *}$ atomic number densities with respect to oxygen, see Table 9.

considerations in Sect. 5.5). This possibility may be fully assessed only by high angular resolution observations, which only new generation observatories such as Herschel are able to perform.

\subsubsection{The [Ne III], [Ar III], [Ne V], [Ar V] and [S III] line flux ratios}

All these lines are detected in the Spitzer data and, because of its much higher angular resolution, provide much more information for the analysis.

The upper limits to the [Ne III] and [Ar III] line ratios (see Table 6 for details on these and other line ratios) do not constrain the plasma parameters much, since both of them correspond to a wide range of densities and temperatures (Rubin et al. 2001).

Limits to the $[\mathrm{Ne} \mathrm{V}$ ] line ratio also do not provide strong constraints partly because of spectral fringing near $20 \mu \mathrm{m}$ in the IRS spectra (Ennis et al. 2006) that increases the effective noise level. Nevertheless, the limit to the [Ne V] $24.32 \mu \mathrm{m}$ line implies that the $[\mathrm{Ne} \mathrm{V}]$ emission mostly or entirely originates in the postshock cooling region.

As for the case of [O III], we derived the fraction of the fine-structure line emission originating in the pre-shock region for [S III] and [Ar V] lines. Most of the [S III] line at $33.48 \mu \mathrm{m}$ originates in the pre-shock region, which we use to estimate the S abundance in Sect. 4.2.

Existence of $\mathrm{Ar}^{4+}$ in the pre-shock region (see Table 6) is understandable, given its relatively low $\mathrm{Ar}^{3+}$ ionization potential of $60 \mathrm{eV}$, comparable to the ionization potential of $\mathrm{O}^{2+}(55 \mathrm{eV})$. Therefore, similar amounts of $\mathrm{O}^{2+}$ and $\mathrm{O}^{3+}$ in the pre-shock photoionized region in the SD-200 model give us a reason to deduce that $\mathrm{Ar}^{3+}$ and $\mathrm{Ar}^{4+}$ ions also have similar number densities. We note that Chevalier \& Kirshner (1979) demonstrated that the visible [Ar IV] lines originate in the low-density environment, which we associate with a pre-shock PIR.

\subsection{Abundances from the flux ratios to the $5007 \AA$ line}

In the general case, ionic and elemental abundances derived from the individual line intensities are strongly dependent on the theoretical model of the fast-moving knots. This model dependence may be minimized if the spectral lines are known to be emitted in the pre-shock or the post-shock region only.

Some dependence on the underlying model still remains, but the model features used in our analysis are relatively robust. For example, the peak of the post-shock plasma emission measure around $10^{4} \mathrm{~K}$ is determined by the cooling rate that slows down at $2 \times 10^{4} \mathrm{~K}$ as resonance ultraviolet lines of various ions stop being effectively excited. The low-temperature limit of the lineemitting region is computed from the upper transition level excitation energy, which is a simple but reliable estimate.

The ionic abundances in the pre-shock region are stronger dependent on the underlying model, since we estimate them for all elements from average ionization potential of the oxygen ions in the SD-200 model. Thus, the pre-shock abundances may be incorrect by up to a factor of a several.

As follows from the theoretical models, spectral lines of singly ionized species (e.g., [Ar II], [Si II], etc.) arise mostly in the photoionized regions, whereas ones of highly-ionized species (e.g., [Ne V], [Mg V], etc.) arise mostly in the post-shock cooling regions. The only exception is the [ArV] line, but the pre-shock region contribution to its lines have already been estimated above.

Below we discuss these two line groups separately and summarize the results in Tables 7 and 8 .

Because of extreme chemical inhomogeneities in the Cas A supernova remnant, one should not expect abundances in X-ray emitting low-density ejecta to be the same as in much denser optically-emitting knots. In our studies of infrared lines from dense knots, the abundances determined from optical spectra should be more reliable. However, optical abundance precision is not expected to be superior to a factor of two, because of the very approximate physical models used in the data analysis. Optical 
Table 9. Summary of elemental abundance $n_{\text {element }} / n_{\mathrm{O}}$ values in Cas A ejecta from various sources.

\begin{tabular}{lllllll}
\hline \hline & CK78 & CK79 & Wil02 & LH03 & Laz06 & Used in this paper \\
\hline Method & Optical & Optical & X-ray & X-ray & X-ray & - \\
\hline $\mathrm{Ne} / \mathrm{O}$ & 0.025 & $<0.013$ & 0.02 & - & - & 0.02 \\
$\mathrm{Mg} / \mathrm{O}$ & $<0.04$ & $<0.005$ & 0.004 & - & $0.005-0.03$ & 0.005 \\
$\mathrm{Si} / \mathrm{O}$ & - & - & 0.025 & $0.003-0.015$ & $0.008-0.06$ & 0.05 \\
$\mathrm{~S} / \mathrm{O}$ & 0.06 & $0.002-0.7$ & 0.014 & - & $0.006-0.05$ & 0.05 \\
$\mathrm{Ar} / \mathrm{O}$ & 0.004 & $0.003-0.1$ & 0.0035 & - & - & 0.005 \\
$\mathrm{Fe} / \mathrm{O}$ & 0.003 & $<0.001$ & $0.006-0.02$ & $0.002-0.01$ & - & 0.005 \\
\hline
\end{tabular}

The "average" data used in this paper are shown in the last column. Data source abbreviations: Chevalier \& Kirshner (1978) - CK78, Chevalier \& Kirshner (1979) - CK79, Willingale et al. (2002) - Wil02, Laming \& Hwang (2003) - LH03, Lazendic et al. (2006) - Laz06.

data are also not available for Si and are especially uncertain for Fe and Mg (Chevalier \& Kirshner 1978).

For our analysis, we summarized the available data on abundances in the Cas A ejecta (see Table 9) and also used some "average" values. However, as noted above and clearly seen also from Table 9, the observed abundances vary dramatically from place to place.

\subsubsection{High-ionization ionic species from the post-shock cooling region}

An easy estimate of the plasma composition may be made if the problem is simplified by assuming that emission originates in a single layer with given temperature and electron density. In this case one can estimate relative number densities of two types of ions from their line fluxes. The line flux ratio is then expressed from Eq. (2) as

$\frac{I_{1}}{I_{2}}=\frac{v_{1} \varepsilon_{1} n_{\text {ion, } 1} \Delta t_{1}}{v_{2} \varepsilon_{2} n_{\text {ion, } 2} \Delta t_{2}}$,

where $n_{\text {ion }}$ is ionic number density, $\Delta t$ is the line emission duration in the post-shock cooling plasma element, and it is assumed that the electron densities are the same for regions emitting both lines. The line emissivities entering Eq. (5) are computed as described in Sect. 2.2.

We note that the application of Eq. (5) is possible in the SD-200 model because both the ion and electron density remain approximately constant at $T \lesssim 10^{4} \mathrm{~K}$. This, combined with the relatively weak dependence of the fine-structure line emissivity on temperature, allows us to use this simple estimate without introducing errors of more than a factor of two or so.

Equation (5) may be inverted to determine the relative ionic abundances from the observed line ratios. In it, we always assume the second line in the ratio to be the [O III] $5007 \AA$ line.

In the following, we use the SD-200 model with the post-shock $\mathrm{O}^{2+}$ abundance in the line emitting region of $n\left(\mathrm{O}^{2+}\right) / n(\mathrm{O}) \approx 1 \%$. We also take into account that only about $2 \%$ of the total $5007 \AA$ line emission originates in the post-shock plasma according to the SD-200 model. This fraction is uncertain by up to a factor of about two, thus introducing a systematic error of similar magnitude in all post-shock ionic abundance determinations.

There are no published results about the theoretically predicted abundances of ions other than oxygen in the post-shock cooling region of the FMKs. To estimate them, we utilized the electron and ion densities and the cooling function from Sutherland \& Dopita (1995) and traced the ion ionization and recombination processes in the post-shock cooling phase using atomic data compiled by Dima Verner ${ }^{10}$ for the Cloudy code (Ferland et al. 1998).

Another source of uncertainty in the ionic abundance determination using our method are the model-dependent values of $\Delta t$, which were also computed by tracking the plasma cooling and recombination. However, calculations show that for different ions and lines the $\Delta t$ values differ by no more than approximately a factor of two, as determined by the general emission measure behavior. The resulting model abundances are compared with the observational results in Table 7 .

It is seen that the abundances inferred from the SD-200 model seem to be generally consistent with the results of observations, showing that the model in general correctly represents the temperature and density evolution in the post-shock cooling region. The $[\mathrm{ArV}]$ and $[\mathrm{Ne} \mathrm{V}]$ lines are the only ones showing significant differences from the model expectations, although the derived ionic abundances do not significantly exceed the corresponding elemental abundances.

We note that the two highest ionization fine-structure lines of [Si X] and [Si VI] observed from the fast-moving knots have not been observed by Spitzer, but detected in the near-infrared spectra by Gerardy \& Fesen (2001). We determined their line flux ratios to the [O III] $5007 \AA$ line using reddening measurements from the same regions by Hurford \& Fesen (1996).

\subsubsection{Low-ionization ionic species from the pre-shock PIR}

The post-shock plasma recombination is significantly slower than the cooling and, even at $T_{\mathrm{e}} \approx 300 \mathrm{~K}$, the post-shock cooling plasma still mostly contains multiply-charged ions, resulting in essentially no infrared line emission from singly ionized atoms at wavelengths shorter than several tens of $\mu \mathrm{m}$.

In this section, we neglect any contribution to the line fluxes from the post-shock photoionized region. Its possible existence and physical conditions are discussed in Sect. 5.

We can then write Eq. (2) in a form similar to Eq. (5) for the pre-shock region given by

$\frac{I_{1}}{I_{2}}=\frac{v_{1} \varepsilon_{1} n_{\mathrm{e}, 1} n_{\text {ion, } 1} \Delta t_{1}}{v_{2} \varepsilon_{2} n_{\mathrm{e}, 2} n_{\mathrm{ion}, 2} \Delta t_{2}}$

The equation also contains the electron density ratio, because ions of different ionization potentials exist in regions of different electron densities.

In contrast to the post-shock case, here the timescales $\Delta t$ are determined by the ionization rates and may vary by an order of magnitude or more from ion to ion.

From the SD-200 model, we adopt the average ionization potential as a function of time and compute the ionization state

${ }^{10}$ http://www.pa.uky.edu/ verner/atom.html 


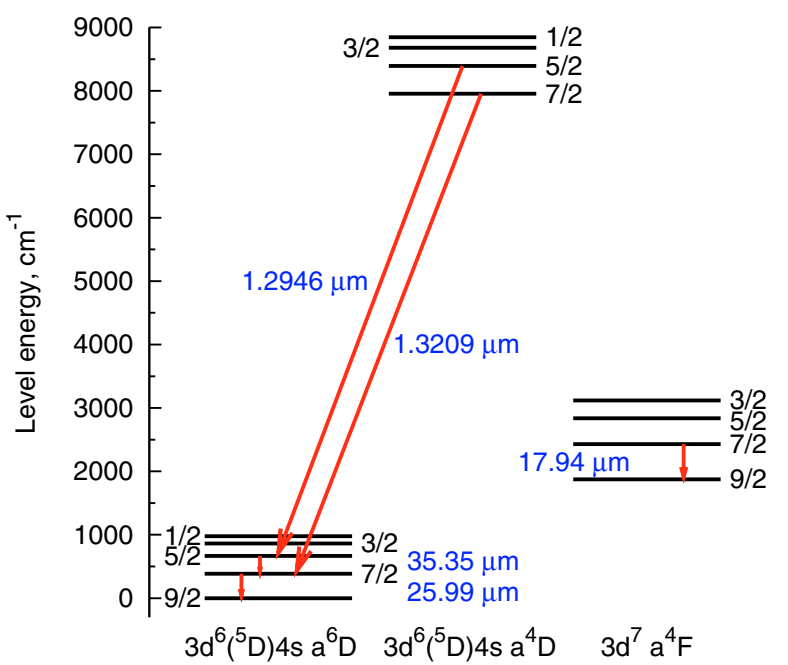

Fig. 9. Energy diagram of the lowest levels of $\mathrm{Fe}^{+}$ion with transitions used in our analysis and corresponding line wavelengths. The electron configurations are given below the diagram; numbers besides the levels denote total angular momentum $J$. Data from Ralchenko et al. (2007).

distribution of all elements with the simple approximation that this average ionization potential is equal for all of them. We also take the temperature profile from the model, which has some effect on the resulting emissivities. Fortunately, precise temperature values have only a minor effect on the obtained abundances, since the infrared line emissivities depend weakly on $T_{\mathrm{e}}$ above $10^{3} \mathrm{~K}$ or so.

In the pre-shock ionization front of the SD-200 model, each initially neutral atom is consequently ionized with maximum ionic abundance of each ion of the order of $50-100 \%$. Therefore, the ionic abundances obtained in Table 8 should be quite close to the elemental abundances, provided that the $\Delta t$ values are estimated correctly and the pre-shock PIR provides the major contribution to the line intensity ${ }^{11}$.

As a test case, we also included in Table 8 the similarly computed $\mathrm{O}^{2+}$ abundance from the [O III] $88.36 \mu \mathrm{m}$ line. The result is very close to the expected value of one (even too close, given our uncertainties of about a factor of two), illustrating the reliability of our method.

Although there are several [Fe II] spectral lines in the infrared spectral range probed by Spitzer that originate in the low-lying electronic configurations, the two most promising are blended with other stronger lines (one at $25.99 \mu \mathrm{m}$ is blended with the [O IV] line at $25.91 \mu \mathrm{m}$ and one at $35.35 \mu \mathrm{m}$ is blended with [Si II] line at $34.81 \mu \mathrm{m}$ ). Therefore in the Spitzer wavelength range, we used for our analysis only the [Fe II] line at $17.94 \mu \mathrm{m}$ (see Fig. 9 for the energy levels of $\mathrm{Fe}^{+}$corresponding to these transitions).

From Table 8, it is seen that the $\mathrm{Ar}^{+}, \mathrm{Si}^{+}$, and $\mathrm{Fe}^{+}$abundances estimated assuming that their fine-structure lines arise exclusively in the pre-shock region are much higher than the corresponding elemental abundance values obtained from optical and X-ray data (Chevalier \& Kirshner 1979; Willingale et al. 2002). The possible solutions of this discrepancy are discussed in Sect. 5.1 .

\footnotetext{
11 Abundance of $\mathrm{Ar}^{4+}$ estimated from [ArV] lines is much lower than the Ar elemental abundance, presumably because only some fraction of $\mathrm{Ar}$ atoms is ionized as far as $\mathrm{Ar}^{4+}$ in the pre-shock region.
}

\section{Post-shock photoionized region}

\subsection{On the too high pre-shock intensities of some lines}

Abundances of $\mathrm{Ne}$ and $\mathrm{S}$ derived assuming that the fine-structure lines of [Ne II] and [S III] are emitted in the pre-shock photoionized region of the SD-200 model agree reasonably well with the results of optical and X-ray observations. Simultaneously, similarly derived abundances of other singly-charged ions $\left(\mathrm{Si}^{+}, \mathrm{Ar}^{+}\right.$, $\mathrm{Fe}^{+}$) are unreasonably high. They are even much higher than their estimated elemental abundances (compare with Table 9).

Within the frame of the SD-200 model, it is difficult to interpret these results and at the same time to comment on the absence of some other infrared lines, such as [Fe III] line at $22.93 \mu \mathrm{m}$ and many others.

There are several possible solutions to this mismatch between the model predictions and observations.

- The inconsistency might result from the application of the SD-200 model, describing the plasma dominated by C, O, $\mathrm{Ne}$, and $\mathrm{Mg}$, to the plasma, containing considerable amounts of S, Si, and Ar. However, the total abundance of these elements with respect to oxygen reaches only about $10 \%$, which should not significantly alter the shock structure.

- Flux in the Si and Fe infrared lines might mostly originate in physically separated Fe-dominated clouds. This hypothesis is based on two observations: (a) in [Fe II] line observations Cas A has a different morphology than in the [O IV] line (Ennis et al. 2006; Rho et al. 2008); and (b) X-ray Chandra observations indicate that hot $\mathrm{Si}$ - and $\mathrm{Fe}$-dominated blobs exist in Cas A.

However, the Cas A morphology in the $\mathrm{Fe} \mathrm{K} \alpha$ X-ray line also has a strongly different morphology from that in the [Fe II] $17.94 \mu \mathrm{m}$ line weakening these arguments. This hypothesis could be tested by observations of the Cas A ejecta with higher spectral resolution, which would allow one to determine whether [Fe II] and other infrared lines from the same knots have the same peculiar velocities.

- The [Si II], [Ar II], and [Fe II] lines might originate in separate weakly-ionized photoionized region of high emission measure. It might be situated either before the shock (the "far precursor" region of Itoh 1981a,b) or after it (the postshock PIR present in models of Itoh 1981a,b; Borkowski \& Shull 1990). This hypothesis is discussed further below.

It is possible to distinguish between lines emitted by the preshock and post-shock PIRs because of their strongly different electron densities. For this purpose, we use additional constraints on this line-emitting region that can be obtained from the Gerardy \& Fesen (2001) observations of the near-infrared [Fe II] lines (see Table 6).

The [Fe II] line ratio $I(1.3209 \mu \mathrm{m}) / I(1.2946 \mu \mathrm{m})<1$ (see Fig. 9 for the transition diagram between the $\mathrm{Fe}^{+}$levels) strongly indicates the electron density above $10^{5} \mathrm{~cm}^{-3}$ at any temperature, thus weakening the hypothesis of the "far precursor". In the case of lower densities, this ratio is always higher, reaching values of 10-20 in the low-density limit because of strong differences in the excitation rates of the respective transition upper levels (Ramsbottom et al. 2007).

Another problem for the pre-shock "far precursor" hypothesis is the observed ratio of the [Si II] $34.81 \mu \mathrm{m}$ to the [Fe II] $17.94 \mu \mathrm{m}$ lines of about 2. Both neutral and singly ionized $\mathrm{Si}$ and $\mathrm{Fe}$ atoms have similar ionization potentials, therefore we might expect that their ionization fractions are similar. From the line emissivities for the low-density conditions and average abundance ratio of $n(\mathrm{Si}) / n(\mathrm{Fe}) \approx 10$ (Table 9), the expected line ratio 
Table 10. Spectral lines used for analysis of the post-shock photoionized region. (See text for details).

\begin{tabular}{|c|c|c|c|c|c|}
\hline Spectrum & $\lambda$ & $I / I(5007 \AA)$ & Source & Observed? & Application \\
\hline He I & $10830 \AA$ & $0.006^{*}$ & GF01 & Yes? & He abundance \\
\hline$[\mathrm{C} \mathrm{I}]$ & $8727 \AA$ & 0.002 & HF96 & Yes & Post-shock PIR disruption \\
\hline \multirow[t]{3}{*}[\mathrm{O}I]{} & $6300 \AA$ & 0.08 & HF96 & Yes & C abundance \\
\hline & $63.19 \mu \mathrm{m}$ & 0.07 & This paper & Yes & $\begin{array}{l}\text { O ionization state, electron density, } \\
\text { extent of the post-shock PIR }\end{array}$ \\
\hline & $145.5 \mu \mathrm{m}$ & $<0.0024$ & This paper & No & Electron density \\
\hline O I & $7774 \AA$ & 0.003 & HF96 & Yes & $\begin{array}{l}\text { Extent of the post-shock PIR, O ionization state, } \\
\text { reference for line ratios }\end{array}$ \\
\hline [Si II $]$ & $34.81 \mu \mathrm{m}$ & 0.07 & This paper & Yes & Extent of the post-shock PIR \\
\hline [S I $]$ & $25.25 \mu \mathrm{m}$ & $<0.01$ & This paper & No & $\mathrm{S}^{0}$ abundance \\
\hline [S II $]$ & $10287-10370 \AA$ & 0.11 & HF96, GF01 & Yes & Probable multi- $T$ structure of the post-shock PIR \\
\hline [Ar II] & $6.985 \mu \mathrm{m}$ & 0.5 & This paper & Yes & Extent of the post-shock PIR, Ar ionization state \\
\hline \multirow[t]{4}{*}[\mathrm{Fe}\mathrm{II}]{} & $1.2946 \mu \mathrm{m}$ & 0.001 & GF01 & Yes & Electron density \\
\hline & $1.3209 \mu \mathrm{m}$ & 0.0007 & GF01 & Yes & Electron density and temperature \\
\hline & $17.94 \mu \mathrm{m}$ & 0.03 & This paper & Yes & Electron temperature, extent of the post-shock PIR \\
\hline & $25.99 \mu \mathrm{m}$ & $<0.15$ & This paper & No? & Electron temperature \\
\hline
\end{tabular}

${ }^{*}$ An upper limit to the line intensity intensity (blended lines).

Note: references: HF96 - Hurford \& Fesen (1996), GF01 - Gerardy \& Fesen (2001).

is as high as 100 . We therefore conclude that the PIR contributing to the emission in these lines is likely to extend after the shock.

\subsection{Lines originating in the post-shock PIR}

All spectral lines of the neutral, singly-, and doubly-charged ions originate in the pre-shock and the post-shock photoionized regions. The strongly different densities of these regions (by a factor of more than $10^{3}$ ) produces a much lower ionization in the denser post-shock PIR, which is only partially ionized (e.g., Borkowski \& Shull 1990).

In contrast, neutral and singly-ionized atoms are not abundant in the pre-shock PIR, where they are rapidly ionized to higher degrees. Estimates based on the SD-200 model confirm that for these atoms the pre-shock PIR contribution to flux in most of their lines is below 10-30\%.

For example, the [S II] lines close to $6720 \AA$ are strongly damped in the high-density medium of the post-shock PIR and are obviously emitted before the shock. It provides the possibility of evaluating directly the pre-shock contribution to the bright near-infrared [S II] lines near $10300 \AA$ from the low-density line ratios, which turns out to be about $10 \%$.

For our qualitative analysis of the post-shock photoionized region, presented below, we use the lines listed in Table 10 . There are also other lines that originate in the same region, but their analysis does not yield additional conclusions.

\subsection{Charge exchange processes in post-shock PIR}

Atomic and ionic spectral lines in a rarefied partially photoionized cold plasma are produced by

- collisional excitation from the ground state;

- radiative recombination onto excited states, and;

- charge exchange reactions into excited states.

The charge exchange process at low temperatures is particularly effective in our case of partially ionized plasma, as the atomion charge exchange is not slowed by electrical repulsion of the reactants. Since the oxygen atom ionization potential is higher than those of $\mathrm{C}, \mathrm{Si}, \mathrm{S}$, and $\mathrm{Fe}$, the charge exchange reactions may neutralize oxygen simultaneously ionizing these elements.

It is interesting to note that the charge exchange process have never before been accounted for in the theoretical FMK models. The pure-oxygen FMK models (Itoh 1981a,b; Borkowski \& Shull 1990) do not even mention charge exchange between, e.g., neutral and doubly ionized oxygen as one of the relevant processes. Based on the method of Dopita et al. (1984), the Sutherland \& Dopita (1995) computations do not include the charge exchange reactions because the plasma temperature is too low for them to occur, according to Hasted (1962). However, their argument does not apply, since in our case there are pseudocrossings of intermittent molecular ion potential energy curves.

We base our estimates of the rates of the relevant charge exchange reactions on the simple procedure suggested by Pequignot \& Aldrovandi (1986) accounting for considerations of Stancil (2001). Our estimates are presented in Table 11 with the spectral lines produced by a charge exchange into excited states (so-called charge exchange excitation, see, e.g., Dalgarno \& Sternberg 1982; Dalgarno 1985).

Since the charge exchange is by far the most rapid of collisional processes, the ionization state of various elements in the post-shock PIR is insensitive to the charge exchange rate values. Taking these rates lower by one order of magnitude produces only an insignificant change in the ionization states (see Fig. 10 for example). Roughly, the number of ionized oxygen atoms decreases by the total number of $\mathrm{C}, \mathrm{Si}, \mathrm{S}$, and $\mathrm{Fe}$ atoms, provided that $n_{\mathrm{O}}>n_{\mathrm{C}}+n_{\mathrm{Si}}+n_{\mathrm{S}}+n_{\mathrm{Fe}}$.

The charge exchange reactions neutralizing singly charged ions of neon and argon in collisions with atoms of oxygen is estimated to be very slow ${ }^{12}$ (of the order of $1 \times 10^{-13} \mathrm{~cm}^{3} / \mathrm{s}$, Liu \& Dalgarno 1996), therefore the argon and neon ionization state in the post-shock PIR is controlled by ionization and recombination processes. Charge exchange of $\mathrm{Ne}$ and $\mathrm{Ar}$ ions with other neutral atoms $(\mathrm{C}, \mathrm{Si}, \mathrm{S}, \mathrm{Fe})$ is not important since these elements are mostly ionized (see above). This qualitatively explains the strong observed [O I] line at $63.19 \mu \mathrm{m}$ and the absence of fine-structure [Si I], [S I], and [Fe I] infrared lines.

\footnotetext{
12 We are grateful to the anonymous referee for pointing out the source of the $\mathrm{Ne}$ and Ar charge exchange rates with oxygen.
} 
D. Docenko and R. A. Sunyaev: Fine-structure infrared lines from the Cassiopeia A knots

Table 11. Charge exchange reaction rates relevant to the post-shock PIR in an oxygen-dominated medium.

\begin{tabular}{lllll}
\hline \hline Reaction & $\Delta E, \mathrm{eV}$ & $\delta E, \mathrm{eV}$ & $\alpha_{\mathrm{CX}}, \mathrm{cm}^{3} / \mathrm{s}$ & Emitted lines; notes \\
\hline $\mathrm{O}^{+}+\mathrm{C}^{0} \rightarrow \mathrm{O}^{0}\left({ }^{1} \mathrm{D}\right)+\mathrm{C}^{+}$ & 2.36 & 0.39 & $1 \times 10^{-9}$ & [O I $]$ 6300, 6363 \\
$\mathrm{O}^{+}+\mathrm{Si}^{0} \rightarrow \mathrm{O}^{0}+\mathrm{Si}^{+}\left(3 \mathrm{~s} 3 \mathrm{p}^{2}{ }^{4} \mathrm{P}\right)$ & 5.47 & 0.15 & $3 \times 10^{-9}$ & {$[\mathrm{Si} \mathrm{II}] 2329-2351 \AA$} \\
$\mathrm{O}^{+}+\mathrm{S}^{0} \rightarrow \mathrm{O}^{0}+\mathrm{S}^{+}\left({ }^{2} \mathrm{P}\right)$ & 3.26 & 0.22 & $3 \times 10^{-9}$ & {$[\mathrm{~S} \mathrm{II}]$ 4069, 4076, 6716, 6731, 10287-10 370 } \\
$\mathrm{O}^{+}+\mathrm{Fe}^{0} \rightarrow \mathrm{O}^{0}+\mathrm{Fe}^{+}$(excited) & 5.72 & many transitions & $1.7 \times 10^{-9}$ & multiple lines; rate from Rutherford \& Vroom (1972) \\
\hline
\end{tabular}

Notes: it is assumed that the atoms and ions are in their ground states (or excited fine-structure states) before the charge-exchange reaction. $\Delta E$ denotes the difference in the ionization potentials of atom and ion in their ground states, $\delta E$ denotes the same difference for outgoing atom and ion in specified states, and $\alpha_{\mathrm{CX}}$ is the charge exchange rate estimate. Only excited state classification is shown.

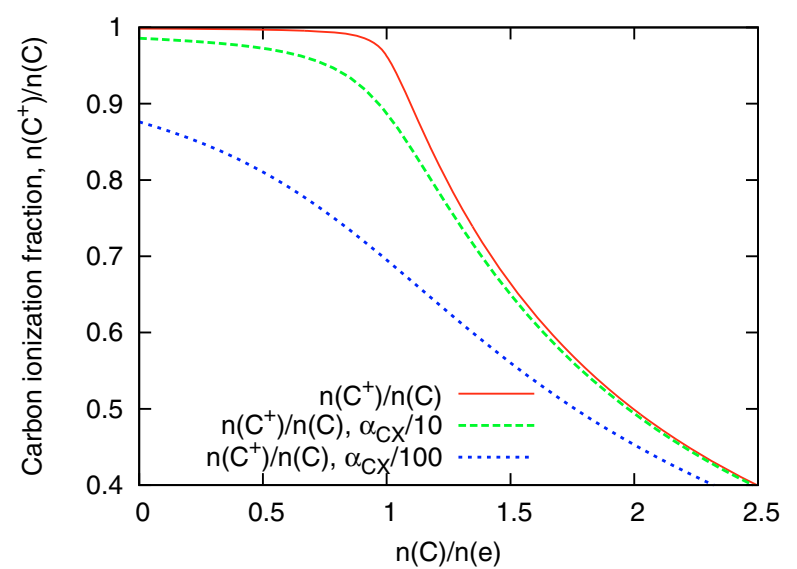

Fig. 10. Ionization state of carbon in an oxygen-carbon partially photoionized plasma. Since carbon and oxygen photoionization and recombination rates are similar, the carbon ionization fraction is almost exclusively dependent on the ratio $n_{\mathrm{C}} / n_{\mathrm{e}}$. Dependencies on both total atomic density and carbon abundance at constant $n_{\mathrm{e}}$ are minor. The upper curve corresponds to the charge exchange rate given in Table 11, and the middle and lower curves to the rates artificially diminished by factors of 10 and 100. It is seen that the charge exchange processes result in carbon being ionized by more than 95-99\% when the number density of carbon atoms and ions is less than that of electrons.

The weakness of the neutral oxygen recombination line at $7774 \AA$ is also indirect proof of the charge exchange process operating in the post-shock PIR ${ }^{13}$. The $7774 \AA$ line is traditionally used to estimate the extent of this region (Itoh 1986), as the line intensity is proportional to the amount of oxygen recombination (in the absence of charge exchange, also to ionization) events, which depends on the optical depth of the post-shock PIR. At the post-shock PIR temperatures, each radiative recombination of a singly ionized oxygen atom is followed by the $7774 \AA$ line emission with a probability of about $p=0.4$ (Pequignot \& Aldrovandi 1986).

However, since ionized oxygen in the post-shock PIR may be neutralized by both recombination and charge exchange, the $7774 \AA$ line weakens, and its flux alone does not provide enough data to determine the optical depth of the post-shock PIR. To obtain full information about the number of recombinations in the post-shock PIR plasma, deeper observations are needed that would also detect optical and near-infrared recombination lines of other elements (e.g., C I line at $10695 \AA$, S I line at $9237 \AA$, etc.).

However, a lot of useful information may be extracted from the flux of the $7774 \AA$ recombination line. For example, one can

\footnotetext{
13 We are grateful to the anonymous referee for pointing out the usefulness of the O I $7774 \AA$ spectral line.
}

roughly estimate the oxygen ionization from the ratio of its flux to the total flux from the post-shock PIR.

If plasma contains only oxygen, then the number of $\mathrm{O}$ recombination events will be equal to the number of absorbed ionizing photons. The presence of other elements with similar ionization cross-sections and recombination rates will decrease the number of oxygen recombinations by a factor $n_{\mathrm{O}} / n_{\mathrm{t}}$, if $n_{\mathrm{t}}$ remains constant and no charge exchange reactions occur. In this case, the ratio of the $7774 \AA$ line flux to the total flux emitted from the post-shock PIR cannot be smaller than

$$
\frac{I(7774 \AA)}{\sum I}=\frac{p h v_{7774}}{h v_{\text {ioniz }}} \frac{n_{\mathrm{O}}}{n_{\mathrm{t}}}=\frac{0.4 \times 1.6 \mathrm{eV}}{27 \mathrm{eV}} \frac{n_{\mathrm{O}}}{n_{\mathrm{t}}}=0.024 \frac{n_{\mathrm{O}}}{n_{\mathrm{t}}},
$$

where $h v_{\text {ioniz }}$ is the average ionizing photon energy. It is estimated to be

$h v_{\text {ioniz }}=\frac{1}{2} \times \frac{H_{\mathrm{sh}}}{N_{\mathrm{ph}}}=\frac{1}{2} \times \frac{(5 / 2) k_{\mathrm{B}} T_{\mathrm{sh}} \times\left(n_{\mathrm{e}} / n_{\mathrm{t}}+1\right) n_{\mathrm{t}} / n_{\mathrm{O}}}{N_{\mathrm{ph}}}$,

where $T_{\mathrm{sh}}$ and $n_{\mathrm{e}} / n_{\mathrm{t}}$ are the temperature and the electron-toatom ratio at the shock front, respectively, $H_{\mathrm{sh}} \approx 2250 \mathrm{eV}$ is the enthalpy per oxygen atom at the shock front (the cooling postshock plasma should be isobaric most of the temperature range), and $N_{\mathrm{ph}} \approx 42$ is the average number of emitted ionizing photons per oxygen atom (its value being derived from the ionization parameter $Q$ of Sutherland \& Dopita 1995) in the SD-200 model. The factor of $1 / 2$ is the fraction of downstream photons.

However, the observed value of this $7774 \AA$ line ratio is at least a factor of three lower (its ratio with respect to the FS line of [Ar II] alone is about 0.007 , see Tables 10 and 5). We note that this ratio of $1 / 3$ is an absolute upper limit. The ratio of the expected $7774 \AA$ line flux to the observed one may be much higher, since part of the much stronger dust continuum emission (of flux 10-40 times higher than that in the $5007 \AA$ line) may originate in the post-shock PIR.

From this, we infer that at least two thirds of the ionized oxygen atoms recombine by means of charge exchange. Assuming additionally that:

- this results in a complete ionization of atoms of other elements connected to oxygen by charge exchange reactions (see above);

- the recombination rates of all singly ionized atoms are similar, and;

- the average ionization degree of plasma is about $40 \%$ (Borkowski \& Shull 1990),

we find that the total number of elements related to oxygen by charge exchange reactions constitutes about $2 / 3$ of the number density of oxygen itself (i.e., $n_{\mathrm{O}} / n_{\mathrm{t}} \approx 0.6$ ) and the oxygen ionization $n\left(\mathrm{O}^{+}\right) / n(\mathrm{O})$ is not higher than $0.10-0.15$. 


\subsection{The post-shock PIR temperature and density}

Once the physical situation has been established, we now determine the conditions in the post-shock photoionized region.

The lower limit to the temperature of about $1000 \mathrm{~K}$ is obtained from the requirement that the [Fe II] $25.99 \mu \mathrm{m}$ line emitted by this region should not be too bright (an observational constraint is $I(25.99 \mu \mathrm{m}) / I(17.94 \mu \mathrm{m})<5$, see Sect. 3.2.1).

The very low value of the near-infrared [Fe II] $1.3209 \mu \mathrm{m}$ line ratio to the $17.94 \mu \mathrm{m}$ line corresponds to $T_{\mathrm{e}} \approx 1700 \mathrm{~K}$ in the high-density limit (the high electron density follows from the [O I] fine-structure line ratio, see Sect. 4.1.2). This [Fe II] line ratio is very sensitive to the temperature because of the different excitation potentials of the transitions upper levels and possibly provides only an upper limit to the temperature with the nearinfrared $1.3209 \mu \mathrm{m}$ line partially arising in some smaller and hotter region.

Assuming in our analysis the PIR temperature of $1500 \mathrm{~K}$, we can obtain stronger constraints on the electron density from the limit on the FIR [OI] line ratio, assuming that these lines originate in the post-shock PIR, as discussed in Sect. 5.5.

In this case, the FIR [OI] line ratio corresponds to $n_{\mathrm{e}} \gtrsim$ $5 \times 10^{5} \mathrm{~cm}^{-3}$. We assume this value for the electron density and twice as large a value $n_{\mathrm{t}}=1 \times 10^{6} \mathrm{~cm}^{-3}$ for the total atom and ion number density (average ionization of about $40-50 \%$ is obtained by Borkowski \& Shull (1990) and should be approximately correct, since recombination rates of all singly charged ions are similar).

We note that based on the assumption of constant post-shock gas pressure this $n_{\mathrm{t}}$ value corresponds to the pre-shock number density of about one hundred atoms per $\mathrm{cm}^{3}$, implying that the magnetic field does not significantly modify the post-shock plasma compression.

\subsection{The post-shock PIR ionization and thickness}

The ratio of the neutral oxygen lines $I(7774 \AA) / I(63.19 \mu \mathrm{m}) \approx$ 0.045 may be used to infer the oxygen ionization degree, which is found to be $n\left(\mathrm{O}^{+}\right) / n\left(\mathrm{O}^{0}\right) \approx 0.06$ for our assumed electron density. This estimate is precise up to a factor of two if $n_{\mathrm{e}}$ is in the range between $1 \times 10^{5}$ and $1 \times 10^{6} \mathrm{~cm}^{-3}$ and only weakly depends on the region temperature.

The value of the oxygen ionization degree is in reasonable accordance with the estimates of Sect. 5.3. This is also a reason for us to assume that most of the $63.19 \mu \mathrm{m}$ emission originates in the FMKs, and not in some more diffuse regions around them (remember that this line was observed by ISO, which had poor angular resolution). In its turn, this allows one to use the [O I] $63.19 \mu \mathrm{m}$ line intensity to estimate the extent of the post-shock PIR, assuming that it is homogeneous.

To estimate the post-shock PIR thickness (see Fig. 1), we can also use the fine-structure lines of [Si II], [Ar II] and [Fe II], as well as the O I $7774 \AA$ recombination line. These estimates are summarized in Table 12.

Closer agreement between the region sizes obtained from the $[\mathrm{OI}]$ and [Fe II] lines may be achieved if the region temperature is assumed to be about $1800-2000 \mathrm{~K}$ (then the [Fe II] line emissivity will increase decreasing its region size estimate to $\left.(2.0-2.2) \times 10^{11} \mathrm{~cm}\right)$.

The estimate from the $7774 \AA$ line also becomes closer to the value derived from the $[\mathrm{O} \mathrm{I}]$ line at these slightly higher temperatures, becoming about $9.3 \times 10^{10} \mathrm{~cm}$. Alternatively, decreasing the oxygen ionization degree increases the post-shock PIR size estimate obtained from this line.
Table 12. Estimates of the post-shock photoionized region thickness $l_{\text {PSPIR }}$ from several fine-structure line ratios to the $5007 \AA$ line.

\begin{tabular}{|c|c|c|c|}
\hline Spectral line & $\varepsilon, \mathrm{erg} \mathrm{cm}^{3} / \mathrm{s}$ & $n$ (ion) $/ n($ elem $)$ & $l_{\mathrm{PSPIR}}, \mathrm{cm}$ \\
\hline [O I] $63.19 \mu \mathrm{m}$ & $1.2 \times 10^{-24}$ & 0.94 & $1.2 \times 10^{11}$ \\
\hline [Si II] $34.81 \mu \mathrm{m}$ & $1.3 \times 10^{-23}$ & 1.0 & $2.1 \times 10^{11}$ \\
\hline [Ar II] $6.985 \mu \mathrm{m}$ & $2.0 \times 10^{-21}$ & 0.4 & $2.0 \times 10^{11}$ \\
\hline [Fe II] $17.94 \mu \mathrm{m}$ & $4.0 \times 10^{-23}$ & 1.0 & $3.0 \times 10^{11}$ \\
\hline O I $7774 \AA$ & $1.3 \times 10^{-24}$ & 0.06 & $8.0 \times 10^{10}$ \\
\hline
\end{tabular}

Notes: estimates made for post-shock PIR electron density $n_{\mathrm{e}}=5 \times$ $10^{5} \mathrm{~cm}^{-3}$ and temperature $T_{\mathrm{e}}=1500 \mathrm{~K}$. Oxygen abundance have been taken to be $n_{\mathrm{O}} / n_{\mathrm{t}}=0.6$ (see Sect. 5.3), other abundances as in Table 9 .

It is worth noting that values of the post-shock PIR size in Table 12 are only about factor of about two smaller than the value of $3 \times 10^{11} \mathrm{~cm}$ predicted by Borkowski \& Shull (1990). This observational evidence therefore does not require significant truncation of the post-shock region, proposed by Itoh (1986). However, other spectroscopic signatures, discussed in Sect. 5.9, show that some truncation of the post-shock PIR seems to be present.

\subsection{Amount of carbon in the FMKs}

The [OI] $6300 \AA$ line originates in neither the pre-shock PIR, nor the post-shock cooling region, as shown by Sutherland \& Dopita (1995), therefore its source is located in the post-shock PIR. Since it is a factor of 25 stronger than the O I $7774 \AA$ A recombination line, we can conclude that recombination provides only a minor contribution to the line flux. It may be produced by collisional excitation, but then the corresponding temperature from the $I(6300 \AA) / I(63.19 \mu \mathrm{m})$ line ratio of about unity corresponds to temperatures of at least $3000 \mathrm{~K}$.

However, the $6300 \AA$ line may be produced by a charge exchange reaction with carbon ${ }^{14}$ (see Table 11). As this reaction is very fast, almost all the carbon is ionized and every carbon recombination will be immediately followed by the charge exchange reaction with an oxygen ion. Then, with a probability of about $72 \%$ (accounting for collisional transitions downwards), oxygen emits the $6300 \AA$ line. A rather strong observed $6300 \AA$ line infers a considerably high abundance of carbon in the FMK plasma.

Specifically, from this line ratio to the $7774 \AA$ line, one can conclude that the carbon abundance is

$n_{\mathrm{C}} / n_{\mathrm{O}} \approx 0.5$

assuming that all carbon is ionized.

This result is only weakly dependent on the post-shock PIR temperature, as long as it stays below $3000 \mathrm{~K}$, where collisional excitation of $6300 \AA$ begins to play a role, and the electron density, as long as it is lower than about $5 \times 10^{6} \mathrm{~cm}^{-3}$, where collisional de-excitation in neutral oxygen starts to diminish the $6300 \AA$ line emissivity. However, estimated carbon abundance is proportional to the assumed oxygen ionization, taken here to be 0.06 (see Sect. 5.5) and is therefore uncertain by up to a factor of two.

\footnotetext{
${ }^{14}$ Note that the charge exchange with other elements do not produce the $6300 \AA$ line; carbon is unique in this respect.
} 


\subsection{Sulfur in the post-shock PIR}

From the non-detection of the $[\mathrm{SI}]$ fine structure line at $25.25 \mu \mathrm{m}$, we can define an upper limit to the neutral sulfur abundance in the post-shock PIR. For this purpose, we compare the [S I] $25.25 \mu \mathrm{m}$ line with the [O I] $63.19 \mu \mathrm{m}$ line having similar dependence on temperature and density. The upper limit on their ratio $I(25.25 \mu \mathrm{m}) / I(63.19 \mu \mathrm{m})<0.14$ corresponds to the abundance ratio of $n\left(\mathrm{~S}^{0}\right) / n\left(\mathrm{O}^{0}\right)<0.004$, showing that sulfur is more than $90 \%$ ionized.

We note that in the framework of our model it is difficult to understand the brightness of the [S II] lines near $10300 \AA$. As discussed in Sect. 5.2, they are emitted after the shock. However, in our simple single-temperature model none of the line production mechanisms - radiative recombination, charge exchange with oxygen (see Table 11), or collisional excitation - can explain the high brightness of these lines.

\subsection{Helium in the optical FMKs}

$\mathrm{X}$-ray data infers that a large amount of elements with $Z<8$ are present in the hot Cas A ejecta (Hughes et al. 2000). Obviously, these elements are hydrogen, helium or carbon. Dewey et al. (2007) even suggested that hot X-ray emitting plasma consists of more than $95 \% \mathrm{He}$. This is also consistent with the suggestion by Sutherland \& Dopita (1995) that a hot plasma between the optical FMKs would be much brighter in X-rays than observed, if it consisted mostly of oxygen.

The amount of helium in the FMK plasma may be constrained, e.g., from the upper limit to its well-known nearinfrared recombination line at $10830 \AA$. Each singly charged helium ion recombination with a probability of about $14 \%$ results in the emission of this line (Pequignot et al. 1991). Therefore, the total recombination of helium after the shock will result in $0.14 n(\mathrm{He}) / n(\mathrm{O})$ photons per oxygen atom.

Comparing this with the SD-200 model $5007 \AA$ line intensity of about 7 photons per oxygen atom, we would expect the $I(10830 \AA) / I(5007 \AA) \approx 0.02 n(\mathrm{He}) / n(\mathrm{O})$. The observed line ratio upper limit of 0.006 thus corresponds to the helium abundance upper limit of about $n(\mathrm{He}) / n(\mathrm{O}) \lessgtr 0.3$. This result is consistent with the Chevalier \& Kirshner (1978) and Peimbert \& van den Bergh (1971) estimates, but is 5-10 times more restrictive because of more certain theoretical model of the post-shock PIR.

This value is probably underestimated by up to a factor of two as helium will still be partially ionized in the post-shock PIR and may not recombine fully if this region is disrupted (see below). Nevertheless, it is obvious that the helium abundance of optical FMKs is not very high, implying that optical and X-ray emitting ejecta indeed have different chemical abundances.

\subsection{Evidence of post-shock PIR disruption from spectral lines}

Post-shock region disruption have been observed in both numerical simulations of the shock interaction with the dense interstellar clouds in supernova remnants (Patnaude \& Fesen 2005) and in laser experiments emulating this interaction (Klein et al. 2003).

As shown by Itoh (1986), there are spectroscopic signatures that allow one to determine if the post-shock PIR is disrupted. Above, we have argued that the original diagnostics of Itoh (1986) - the O I $7774 \AA$ line - is not applicable because of the charge exchange between oxygen and other elements, and the poorly constrained typical chemical composition of the FMKs.

Instead we consider other spectral lines that are sensitive to the final recombination in the post-shock PIR. Indeed, if this region is present, then each ion in the post-shock PIR recombines at least once, giving a lower limit to the line intensities due to recombination. The most sensitive to the presence of final recombination are high-excitation lines of atoms that should be completely ionized due to charge exchange with oxygen, i.e., ones that should not be emitted in any other region of a FMK.

As an example, we consider the observed [CI] line at $8727 \AA$. It is emitted with probability of roughly $10 \%$, when a carbon ion recombines. Thus, there should be at least 0.05 photons in this line per every oxygen atom (using the carbon abundance from Sect. 5.6). Comparing this with the SD-200 model $5007 \AA$ line intensity of about 7 photons per oxygen atom, we would expect to measure $I(8727 \AA) / I(5007 \AA) \approx 0.007$. The observed $8727 \AA$ line is a factor of three less intense, implying that not all carbon recombines after the shock.

Unfortunately, this result, which confirms the post-shock PIR disruption, is not very robust, since the carbon abundance value is not very tightly constrained. However, similar diagnostics may be performed with this and other lines using future more sensitive observations. These observations will show us the maximum extent to which each atomic species recombines in the FMKs after the reverse shock.

We note that another argument exists for the post-shock PIR truncation, which is based on energy considerations ${ }^{15}$.The energy of the post-shock PIR line emission per oxygen atom is a factor 5-10 less than the energy that should be deposited if the emission were optically thick to the post-shock cooling plasma emission. The most obvious solution to this problem is postshock PIR truncation.

However, another possibility is that some part of the cooling plasma energy is transferred to dust heating inside the postshock cooling region or post-shock PIR and is then emitted in the infrared dust continuum, which in total emits a factor of 12 more energy than the lines from the post-shock PIR (see Sect. 3.4). Most probably, a combination of these two factors (disruption and continuum emission) explains the apparent difference between the expected and observed post-shock PIR emission-line fluxes.

\subsection{Effects of the dust on the post-shock PIR structure}

Using the data from Rho et al. (2008), it is straightforward to estimate the dust mass in each pixel of the Spitzer map of the Cas A. Its comparison with the optical image of this supernova remnant allows us to determine the dust-to-gas mass ratio in the bright knots from the [O III] line flux predicted by the SD200 model.

If the emitting dust with temperature $T_{\mathrm{d}} \approx 100 \mathrm{~K}$ (Rho et al. 2008 ) is distributed over the entire volume of the knot, then the dust-to-gas mass ratio is of the order of $2-5 \%$. If, on the other hand, the dust emits only in the line-emitting regions, then the dust-to-gas mass ratio is of the order of unity.

If the latter is true, then the current shock models do not apply and construction of an entirely different model is required to describe the shock structure of the Cas A knots. This analysis is outside the scope of this paper.

15 This argument was pointed out by the anonymous referee. 


\section{Recombination lines in the infrared range}

The metal recombination lines (RLs) are another good tracer of the cold ionized plasma. Their emissivities as functions of temperature have no exponential cutoff at low $T_{\mathrm{e}}$ and increase with decreasing temperature approximately as $T_{\mathrm{e}}^{-1}$ at $T_{\mathrm{e}}<10^{4} \mathrm{~K}$. The line emissivities are only weakly dependent on density.

However, their emissivities are 4-6 orders of magnitude less than those of the fine-structure infrared lines at the low-density limit. The infrared RL emissivity ratios to the fine-structure lines increase with electron density due to collisional effects that diminish the fine-structure line emissivities as $n_{\mathrm{e}}^{-1}$ starting from some critical density. At post-shock electron densities of $10^{6} \mathrm{~cm}^{-3}$, the emissivity ratios are much higher (Docenko \& Sunyaev 2008), but the infrared RLs are nevertheless still far less intense than the fine-structure lines.

As two examples, we consider infrared RLs near 10 and $60 \mu \mathrm{m}$. The brightest regions emitting RLs are the post-shock cooling and photoionized regions (due to their high emission measures $n_{e}^{2} l$ ) and cold region between the photoionization front and the shock (due to its extremely low temperature and large extent).

The OI $5 \alpha$ recombination line at $7.45 \mu \mathrm{m}$ originates in the post-shock photoionized region and its strongest component emissivity of $5 \times 10^{-27} \mathrm{erg} \mathrm{cm}^{3} / \mathrm{s}$ is about 200 times less than that of the O I $7774 \AA$ line. The expected recombination line flux is therefore $3 \times 10^{4}$ times less than that of the [Ar II] $6.985 \mu \mathrm{m}$ line, or about $0.5 \%$ of the background continuum emission. However, other elements will also produce recombination lines at the same wavelength (e.g., ionized carbon is almost an order of magnitude more abundant than ionized oxygen, see Sect. 5.6) and the total $5 \alpha$ line flux may be a factor of 10-20 more. Estimates show that this limit is achievable with Spitzer with exposure times of a few hours.

Another example is a O I $11 \alpha \mathrm{RL}$ at $69.03 \mu \mathrm{m}$. Its emissivity is approximately 5000 times less than that of nearby [O I] fine-structure line at $63.19 \mu \mathrm{m}$. Expected intensity, if summing with $11 \alpha$ lines of other ions, is about $5 \times 10^{-15} \mathrm{erg} / \mathrm{cm}^{2} / \mathrm{s}$ from the ISO region \#2. This is approximately equal to the expected $5 \sigma$ 1-hour point source sensitivity of the Herschel PACS instrument (Poglitsch et al. 2006). Since the Cas A is not a point source at the Herschel resolution (PACS has a 10" pixel size and most of the Cas A emission is contained within 4-8 PACS pixels), one needs at least a few hours to achieve the $5 \sigma$ detection of this recombination line.

The recombination lines of highly-charged oxygen ions from the post-shock cooling region are expected to be about an order of magnitude dimmer than the OI RLs because of the lower emission measure in the region emitting these lines.

The metal recombination line observations in the mid- and far-infrared are generally more difficult than in the optical and near-infrared due to lower line emissivities and higher background continuum emission. They are also unable to provide information about the ion producing the spectral line, but only about its electronic charge. However, these observations would allow us to determine abundances of ions residing in cold regions that do not produce fine-structure lines. Special emphasis should be placed on the singly-charged ion lines, since their recombination lines in the optical and near-infrared ranges are divided into many weak components, which makes them more difficult to detect (Docenko \& Sunyaev 2008).

\section{Conclusions}

We have performed an analysis of the supernova remnant Cas A fast-moving knot infrared line intensities, by comparing observations with the predictions of various theoretical models, which describe the FMK emission originating from the reverse shock interaction with the pure-oxygen or oxygen-dominated clouds. For this comparison, we have analyzed archival observational data acquired by the ISO and Spitzer observatories. We conclude that accounting for the electron conductivity is essential to reproducing the observed line ratios. This is the reason why the Borkowski \& Shull (1990) model BS-DC provides the most accurate description of the observed oxygen line relative fluxes, although it is only precise up to a factor of several (the [O I] lines are overpredicted partially because of the too low model pre-shock density).

This emphasizes the need for more sophisticated models describing shock propagation in the oxygen-dominated plasma. In this article, many line flux ratios are derived that will help us to construct these future theoretical models. Future far-infrared and optical observations of higher sensitivity and angular resolution will allow one to obtain far more information about the fast-moving knots once the models are constructed.

Analysis of the infrared lines of $\mathrm{O}, \mathrm{Ne}, \mathrm{Si}, \mathrm{S}, \mathrm{Ar}$, and Fe ions have confirmed the existence of three regions contributing to the infrared and optical line emission:

- Lines of the intermediate- and highly-charged ions that arise in the post-shock rapidly cooling region (e.g., [Ne v], [Ar V], [Si VI], [Si X] lines).

- Singly-charged ions that radiate mostly from the post-shock photo-ionized region (e.g., [O I], [Si II], [Ar II], [Fe II] lines).

- Weakly- and intermediate-charged ion lines originate in the pre-shock photoionized region as well. It gives major contribution to the emission of lines of [Ne II], [O III], [S III], [ArV], etc.

An analysis of the FIR observations jointly with the Hurford \& Fesen (1996) and Gerardy \& Fesen (2001) optical and nearinfrared data has allowed us to construct a consistent model of the post-shock photoionized region. This became possible by accounting for the charge exchange process that diminishes oxygen ionization in the post-shock PIR. Observed high $(>0.9)$ ionization of sulfur and low $(\approx 0.06)$ ionization of oxygen confirms the importance of the charge exchange reactions in this region.

From the observed line flux ratios, it then follows that the post-shock PIR has a temperature $T_{\mathrm{e}} \approx(1500-2000) \mathrm{K}$, electron and ion densities of $n_{\mathrm{e}} \approx 5 \times 10^{5} \mathrm{~cm}^{-3}$ and $n_{\mathrm{t}} \approx 1 \times 10^{6} \mathrm{~cm}^{-3}$ and is approximately $(1-2) \times 10^{11} \mathrm{~cm}$ thick, assuming that oxygen constitutes about $70 \%$ of all atoms in the FMK plasma. Given the uncertainties of the model, this result is consistent with the thickness predicted in the Borkowski \& Shull (1990) model.

We have constrained the helium abundance to be $n(\mathrm{He}) / n(\mathrm{O})<(0.3-0.6)$ and the carbon abundance to be about $n(\mathrm{C}) / n(\mathrm{O}) \approx 0.5$.

However, we recall that the obtained values of elemental abundances and ionization fractions in the post-shock PIR are affected by unknown biases, as our assumptions (plane-shock, single-temperature model) clearly do not adequately represent the structure of this region.

From the comparison of observed infrared line intensities with the theoretically expected values, we conclude that:

- The theoretical models correctly describe the general structure of the FMKs; 
- Accounting for the electron conductivity brings the BS-DC model into far closer agreement with the observed oxygen FIR line ratios to the [O III] $5007 \AA$ line than the BS-F and SD-200 models;

- Accounting for the emission from the photoionized region before the shock front is essential to reproducing the FIR [O III] line ratio;

- The FIR [OI] line intensities in the Itoh (1981b) and Borkowski \& Shull (1990) models are partially overestimated because of the too low pre-shock atom number densities;

- The pre-shock atom number density is at least $100 \mathrm{~cm}^{-3}$ and may be as high as $300 \mathrm{~cm}^{-3}$;

- The high density of the post-shock PIR suggests that there is no significant magnetic-field pressure in this region;

- The post-shock photoionized region truncation, as suggested by Itoh (1986), may be needed to explain the weakness of several lines produced by the final plasma recombination.

The infrared metal recombination lines are shown to be detectable by the planned far-infrared instruments and useful for the derivation of the plasma properties.

The high brightness of the [S II] lines close to $10300 \AA$ remains unexplained by our single-temperature model of the postshock PIR.

Acknowledgements. D.D. is thankful to Mike Revnivtsev for many useful advices on data processing.

The version of the ISO data presented in this paper correspond to the Highly Processed Data Product (HPDP) set called "Uniformly processed LWS L01 spectra" by C. Lloyd, M. Lerate and T. Grundy, available for public use in the ISO Data Archive, http: //www.iso.vilspa.esa.es/ida/.

Some of the data presented in this paper were obtained from the Multimission Archive at the Space Telescope Science Institute (MAST). They are based on observations made with the NASA/ESA Hubble Space Telescope, obtained from the Data Archive at the Space Telescope Science Institute, which is operated by the Association of Universities for Research in Astronomy, Inc., under NASA contract NAS 5-26555. These observations are associated with program \# 10286. Part of this work is based on observations made with the Spitzer Space Telescope, which is operated by the Jet Propulsion Laboratory, California Institute of Technology under a contract with NASA. This research made use of Tiny Tim/Spitzer, developed by John Krist for the Spitzer Science Center. The Center is managed by the California Institute of Technology under a contract with NASA.

CHIANTI is a collaborative project involving the NRL (USA), RAL (UK), MSSL (UK), the Universities of Florence (Italy) and Cambridge (UK), and George Mason University (USA).

\section{Appendix A: Spitzer data cube PSF size estimate}

As described by Smith et al. (2007), the mathematical transformations performed by the CUBISM software change the point spread function (PSF) of the final data cube away from the initial PSF of the Spitzer IRS. This effect should be more pronounced in images with only a few pixels per PSF width, as constructed by the Spitzer IRS data cubes in the second order of each spectrograph module.

To determine the PSF changes induced by the data cube reconstruction, we fit a two-dimensional Gaussian function to the shape of a point-like source 2MASS 23233176+5853204 situated in the map of the SL2 module. This fit indicated that (here $x$ is the coordinate along the individual slits and $y$-across them):

- The source $x$ centroid oscillates with wavelength with an amplitude of about 0.3 pixels. The $y$ centroid remains constant to within 0.05 pixels.

- The PSFs in both the $x$ and $y$ directions are larger than the Spitzer IRS true PSF, computed, e.g., by the stinytim software. The full width at half maximum (FWHM) in the $x$ and $y$ directions of the spectral maps are greater than the IRS true PSF by 0.55 pixels and 0.9 pixels.

The presence of these features is inferred by the CUBISM algorithm description in Smith et al. (2007). Although the values of the increase in PSF dimensions may seem insignificant at first, they are often comparable with the extent of the IRS true PSF (e.g., at $26 \mu \mathrm{m}$, the initial FWHM of the PSF is equal to the 1.2 LL1 module pixel).

Not accounting for the described increase in the PSF size because of processing by the CUBISM software in our case would result in the Spitzer maps being seemingly more diffuse than e.g., optical maps smoothed to the IRS angular resolution.

Although we have measured these effects only in the SL2 module, we assume that the same PSF broadening in pixels occurs in other modules as well. This conclusion is qualitatively confirmed by visual comparison of optical maps smoothed to the corresponding resolution of the data cube spectral line maps.

\section{Appendix B: Derivation of Eq. (4)}

For the approximation of two homogeneous emitting regions 1 and 2 , the observed line $a$ and $b$ flux ratio $R \equiv I_{a} / I_{b}$ may be expressed as

$R \equiv \frac{I_{a}}{I_{b}}=\frac{I_{a, 1}+I_{a, 2}}{I_{b, 1}+I_{b, 2}}=\frac{I_{a, 1}}{I_{b, 1}} \frac{I_{b, 1}}{I_{b, 1}+I_{b, 2}}+\frac{I_{a, 2}}{I_{b, 2}}\left(1-\frac{I_{b, 1}}{I_{b, 1}+I_{b, 2}}\right)$.

Denoting the line flux ratios arising in regions 1 and 2 as $R_{1} \equiv$ $I_{a, 1} / I_{b, 1}$ and $R_{2} \equiv I_{a, 2} / I_{b, 2}$, and introducing the fraction of the total line $b$ emission arising in the region 1 as

$f_{b, 1} \equiv \frac{I_{b, 1}}{I_{b, 1}+I_{b, 2}}$

we obtain

$R=R_{1} f_{b, 1}+R_{2}\left(1-f_{b, 1}\right)$.

Expressing $f_{b, 1}$ from this linear relation, we finally derive Eq. (4):

$f_{b, 1}=\frac{R_{2}-R}{R_{2}-R_{1}}$

\section{References}

Arendt, R. G., Dwek, E., \& Moseley, S. H. 1999, ApJ, 521, 234

Baade, W., \& Minkowski, R. 1954, ApJ, 119, 206

Baars, J. W. M., Genzel, R., Pauliny-Toth, I. I. K., \& et al. 1977, A\&A, 61, 99

Berrington, K. A., Saraph, H. E., \& Tully, J. A. 1998, A\&ASS, 129, 161

Bieging, J. H., \& Crutcher, R. M. 1986, ApJ, 310, 853

Blum, R. D., \& Pradhan, A. K. 1992, ApJS, 80, 425

Borkowski, K. J., \& Shull, J. M. 1990, ApJ, 348, 169

Butler, K., \& Zeippen, C. J. 1994, A\&AS, 108, 1

Chevalier, R. A., \& Kirshner, R. P. 1978, ApJ, 219, 931

Chevalier, R. A., \& Kirshner, R. P. 1979, ApJ, 233, 154

Dalgarno, A. 1985, Nucl. Instr. Meth. Phys. Res. B, 9, 655

Dalgarno, A., \& Sternberg, A. 1982, MNRAS, 200, 77P

Delaney, T. A. 2004, Ph.D. Thesis, University of Minnesota

Dere, K. P., Landi, E., Mason, H. E., Monsignori Fossi, B. C., \& Young, P. R. 1997, A\&ASS, 125, 149

Dewey, D., Delaney, T., \& Lazendic, J. S. 2007, Rev. Mex. Astron. Astrofis. Conf. Ser., 30, 84

Dinerstein, H. L., Lester, D. F., Rank, D. M., Werner, M. W., \& Wooden, D. H. 1987, ApJ, 312, 314

Docenko, D., \& Sunyaev, R. A. 2008, A\&A, 484, 755

Dopita, M. A., Binette, L., \& Tuohy, I. R. 1984, ApJ, 282, 142

Dufton, P. L., \& Kingston, A. E. 1991, MNRAS, 248, 827

Ennis, J. A., Rudnick, L., Reach, W. T., et al. 2006, ApJ, 652, 376 
Ferguson, J. W., Korista, K. T., \& Ferland, G. J. 1997, ApJS, 110, 287 Ferland, G. J., Korista, K. T., Verner, D. A., et al. 1998, Publ. Astron. Soc. Pacific, 110, 761

Fesen, R. A., Hammell, M. C., Morse, J., et al. 2006, ApJ, 645, 283

Galavis, M. E., Mendoza, C., \& Zeippen, C. J. 1995, A\&AS, 111, 347

Gerardy, C. L., \& Fesen, R. A. 2001, Astron. J., 121, 2781

Griffin, D. C., \& Badnell, N. R. 2000, J. Phys. B Atom. Mol. Phys., 33, 4389

Griffin, D. C., Mitnik, D. M., \& Badnell, N. R. 2001, J. Phys. B Atom. Mol Phys., 34, 4401

Gry, C., Swinyard, B., Harwood, A., et al. 2003, The ISO Handbook, Volume III - LWS - The Long Wavelength Spectrometer, Version 2.1, ed. T.G. Mueller,

J. A. D. L. Blommaert, \& P. Garcia-Lario, ESA SP-1262

Hartmann, D. H., Predehl, P., Greiner, J., et al. 1997, Nucl. Phys. A, 621, 83

Hasted, J. B. 1962, in Atomic and Molecular Processes, ed. D. R. Bates, 696

Hines, D. C., Rieke, G. H., Gordon, K. D., et al. 2004, ApJS, 154, 290

Hughes, J. P., Rakowski, C. E., Burrows, D. N., et al. 2000, ApJ, 528, L109

Hurford, A. P., \& Fesen, R. A. 1996, ApJ, 469, 246

Itoh, H. 1981a, Publ. Astron. Soc. Japan, 33, 1

Itoh, H. 1981b, Publ. Astron. Soc. Japan, 33, 521

Itoh, H. 1986, Publ. Astron. Soc. Japan, 38, 717

Kamper, K., \& van den Bergh, S. 1976, ApJS, 32, 351

Kassim, N. E., Perley, R. A., Dwarakanath, K. S., et al. 1995, ApJ, 455, L59

Klein, R. I., Budil, K. S., Perry, T. S., et al. 2003, ApJ., 583, 245

Laming, J. M., \& Hwang, U. 2003, ApJ, 597, 347

Landi, E., Del Zanna, G., Young, P. R., et al. 2006, ApJS, 162, 261

Lazendic, J. S., Dewey, D., Schulz, N. S., et al. 2006, ApJ, 651, 250

Lennon, D. J., \& Burke, V. M. 1994, A\&AS, 103, 273

Liu, W., \& Dalgarno, A. 1996, ApJ, 471, 480

Mazzotta, P., Mazzitelli, G., Colafrancesco, S., et al. A\&AS, 133, 403

Osterbrock, D. E., \& Ferland, G. J. 2006, Astrophysics of Gaseous Nebulae and Active Galactic Nuclei, 2nd. ed. (University Science Books)

Patnaude, D. J., \& Fesen, R. A. 2005, ApJ, 633, 240
Peimbert, M., \& van den Bergh, S. 1971, ApJ, 167, 223

Pelan, J., \& Berrington, K. A. 1995, A\&AS, 110, 209

Pequignot, D., \& Aldrovandi, S. M. V. 1986, A\&A, 161, 169

Pequignot, D., Petitjean, P., \& Boisson, C. 1991, A\&A, 251, 680

Poglitsch, A., Waelkens, C., Bauer, O. H., et al. 2006, in Space Telescopes and Instrumentation I: Optical, Infrared, and Millimeter, ed. J. C. Mather, H. A. MacEwen, M. W. M. de Graauw, Proc. SPIE, 62650B

Ralchenko, Y., Jou, F.-C., Kelleher, D., et al. 2007, National Institute of Standards and Technology, Gaithersburg, MD

Ramsbottom, C. A., Hudson, C. E., Norrington, P. H., et al. 2007, A\&A, 475, 765

Reed, J. E., Hester, J. J., Fabian, A. C., et al. 1995, ApJ, 440, 706

Rho, J., Kozasa, T., Reach, W. T., et al. 2008, ApJ, 673, 271

Riffel, R., Rodríguez-Ardila, A., \& Pastoriza, M. G. 2006, A\&A, 457, 61

Rubin, R. H., Dufour, R. J., Geballe, T. R., et al. 2001, in Spectroscopic Challenges of Photoionized Plasmas, ed. G. Ferland \& D. W. Savin, ASP Conf. Ser., 247, 479

Rutherford, J. A., \& Vroom, D. A. 1972, J. Chem. Phys., 57, 3091

Smith, J. D. T., Armus, L., Dale, D. A., et al. 2007, Publ. Astron. Soc. Pacific, 119,1133

Stancil, P. C. 2001, in Spectroscopic Challenges of Photoionized Plasmas, ed. G. Ferland, \& D. W. Savin, ASP Conf. Ser., 247, 3

Sutherland, R. S., \& Dopita, M. A. 1993, ApJS, 88, 253

Sutherland, R. S., \& Dopita, M. A. 1995, ApJ, 439, 381

Tayal, S. S. 2006, ApJS, 166, 634

Tayal, S. S., \& Gupta, G. P. 1999, ApJ, 526, 544

Thompson, R. I. 1996, ApJ, 459, L61

Willingale, R., Bleeker, J. A. M., van der Heyden, K. J., Kaastra, J. S., \& Vink, J. 2002, A\&A, 381, 1039

Zhang, H. L., Graziani, M., \& Pradhan, A. K. 1994, A\&A, 283, 319

Zombeck, M. 2007, Handbook of Space Astronomy and Astrophysics: Third edn (Cambridge, UK: Cambridge University Press) 